\title{
Uniform multicommodity flows in the hypercube with random edge-capacities
}

\author{
Colin McDiarmid \\ Department of Statistics, \\ University of Oxford, \\ 1 South Parks Road, \\ Oxford, OX1 3TG, UK. \\ cmcd@stats.ox.ac.uk
}

\author{
Alex Scott \\ Mathematical Institute, \\ University of Oxford, \\ Radcliffe Observatory Quarter, \\ Woodstock Road, \\ Oxford, OX2 6GG, UK. \\ scott@maths.ox.ac.uk
}

\author{
Paul Withers \\ Mathematical Institute, \\ University of Oxford, \\ Radcliffe Observatory Quarter, \\ Woodstock Road, \\ Oxford, OX2 6GG, UK. \\ withers@maths.ox.ac.uk
}

March 14, 2016

\begin{abstract}
We give two results for multicommodity flows in the $d$-dimensional hypercube $Q^{d}$ with independent random edge-capacities distributed like a random variable $C$ where $\mathbb{P}[C>0]>1 / 2$. Firstly, with high probability as $d \rightarrow \infty$, the network can support simultaneous multicommodity flows of volume close to $\mathbb{E}[C]$ between all antipodal vertex pairs. Secondly, with high probability, the network can support simultaneous multicommodity flows of volume close to $2^{1-d} \mathbb{E}[C]$ between all vertex pairs. Both results are best possible.
\end{abstract}




\section{Introduction and statement of results}

A network $\mathcal{N}$ consists of an undirected graph together with a capacity $c_{e} \geq 0$ for each edge $e$. Given a collection $\mathcal{V}$ of unordered pairs of vertices in $\mathcal{N}$, a corresponding multicommodity flow $F$ consists of an st-flow $f_{s t}$ for each pair $s, t$ in $\mathcal{V}$. If each $f_{s t}$ has volume $\phi$ we say that $F$ is a uniform multicommodity flow of volume $\phi$. We say that $F$ is feasible if the total flow in each edge $e$ (with no cancellations) is at most its capacity $c_{e}$. The maximum uniform flow volume is the maximum value of $\phi$ such that there is a feasible uniform multicommodity flow of volume $\phi$ in $\mathcal{N}$.

We investigate multicommodity flows in networks with random edgecapacities. Multicommodity flows are of interest in operational research and combinatorial optimisation and sampling (further background can be found later in this section). They have been studied extensively from a "worst-case" perspective, but the "typical" behaviour of multicommodity flow problems is much less well understood. In order to address this, we work with a model in which the underlying graph is fixed and the edge-capacities are random. Aldous, McDiarmid and Scott 44 studied the case in which the underlying graph is complete, the edge-capacities are independent, each distributed like a given random variable $C$, and $\mathcal{V}$ is the collection of all unordered pairs of distinct vertices. They showed that, for a distribution with a finite mean, as $n \rightarrow \infty$ the maximum uniform flow volume converges in probability to a constant $\phi_{*}$, where $\phi_{*}$ is the unique solution to

$$
\mathbb{E}[\max \{C-\phi, 0\}]=2 \mathbb{E}[\max \{\phi-C, 0\}] .
$$

For example $\phi^{*}=\sqrt{2}-1$ when $C$ is uniformly distributed on $[0,1]$. (See [21] for related results on complete multipartite graphs.)

In this paper, we consider another very natural example: the $d$-dimensional (hyper)cube $Q^{d}$, with independent random edge-capacities each distributed like $C$. In this case there are two natural choices for $\mathcal{V}$. When $\mathcal{V}$ is the collection of opposite (or antipodal) pairs we denote the maximum uniform flow volume by $\Phi_{\text {opp }}$. When $\mathcal{V}$ is the collection of all pairs of distinct vertices we use $\Phi_{\text {all }}$.

Finding flows in the cube presents very different problems compared to finding flows in the complete graph. In the complete graph, flow between a pair of vertices that cannot use the direct edge must go at least twice as far, using up at least twice the capacity. Thus the edge-capacity distribution itself is important, as this determines the proportion of flow that can be routed along efficient paths, with other paths being much less efficient. By contrast, the cube is a sparse graph and the distance between a typical pair of vertices is about $d / 2$. This leads to two challenges in finding large-volume 
multicommodity flows: we must show that there are no local obstructions (vertices or small clusters of vertices that are poorly connected to the rest of the graph, so that little flow can escape the region); and, on the larger scale, we must show that most of the flow can be routed along paths of close to optimal length. It turns out that the capacity required for 'local escape' is much less than that required for the efficient flows along long paths: this leads to the limiting optimal flow value depending only on $\mathbb{E}[C]$, which establishes a strong form of a conjecture from [4]. Here are our two main theorems, for $\Phi_{\text {opp }}$ and for $\Phi_{\text {all }}$.

Theorem 1. Let the non-negative random variable $C$ satisfy $\mathbb{E}[C]<\infty$ and $\mathbb{P}[C>0]>1 / 2$. Then, as $d \rightarrow \infty$,

$$
\Phi_{\text {opp }} \rightarrow \mathbb{E}[C] \text { in probability and in expectation. }
$$

Theorem 2. Let the non-negative random variable $C$ satisfy $\mathbb{E}[C]<\infty$ and $\mathbb{P}[C>0]>1 / 2$. Then, as $d \rightarrow \infty$,

$$
2^{d-1} \Phi_{\text {all }} \rightarrow \mathbb{E}[C] \text { in probability and in expectation } .
$$

To find flows as required we need very different proof methods from those used in [4]. Consider Theorem 1. The idea is, for each antipodal pair $s$ and $t$, to allocate flow only to paths of near the minimum length $d$, and to do so as evenly as possible. To achieve this we allocate a natural fraction of the capacity of each edge to this pair: we find that the middle part of the flow can be handled very efficiently; and we can handle the flow near the ends $s$ and $t$ (to achieve 'local escape') by different less efficient methods, if we allow extra capacity, which turns out to be negligible in the limit.

For a non-negative random variable $C$ with $\mathbb{P}[C>0]>\frac{1}{2}$ and $\mathbb{E}[C]=\infty$, it follows directly from these results by truncation that $\Phi_{\text {opp }}$ and $\Phi_{\text {all }}$ both tend to infinity in probability (and so in expectation). Call an edge open when its capacity is $>0$ : and call the network connected when the subgraph formed by the open edges is connected. Clearly $\Phi_{\text {all }}=\Phi_{\text {opp }}=0$ if the network contains an isolated vertex. The condition $\mathbb{P}[C>0]>1 / 2$ is necessary to ensure that, with high probability, the network contains no isolated vertices. Indeed, when $\mathbb{P}(C>0)=1 / 2$, the probability of the network having no isolated vertex $\rightarrow e^{-1}$ as $d \rightarrow \infty$, and the probability that the network is connected tends to the same limit (see [12]).

Plan of the paper. In the remaining part of this section we give some background on multicommodity flows; and in the next section we give formal definitions of such flows and two probabilistic inequalities which are useful later in the paper. The rest of the paper is devoted to proving Theorems 1 
and 2. The convergence in probability in Theorem 1 can be expressed as two parts:

(upper bound) for $\epsilon>0, \quad \mathbb{P}\left[\Phi_{\text {opp }} \leq(1+\epsilon) \mathbb{E}[C]\right] \rightarrow 1$ as $d \rightarrow \infty$

and

(lower bound) for $\epsilon>0, \mathbb{P}\left[\Phi_{\text {opp }} \geq(1-\epsilon) \mathbb{E}[C]\right] \rightarrow 1$ as $d \rightarrow \infty$,

and similarly for Theorem 2. The upper bounds for both theorems are straightforward and are proved in Section 3. Convergence in expectation is also covered in that section. The bulk of the paper is devoted to proving the lower bounds. The lower bound for Theorem 1 is proved in Sections 4 7. and for Theorem 2 in Section 8.

Background on multicommodity flow. Multicommodity flow problems arise in many real-life situations such as flows in transport systems and communication systems, and are studied extensively in Operational Research. See standard texts such as Ahuja, Magnanti and Orlin [1], Chapter 17, and Winston [20], Chapters 7 and 8, for further details. The analysis of multicommodity flows in structured networks that model Markov chains is also useful in establishing bounds on the mixing times for the chains (Sinclair [18]). These can then be used to establish efficient algorithms for the random sampling of combinatorial structures. Such techniques are important in a wide range of problems including, for example, approximating the size of certain sets and combinatorial optimisation by stochastic search. Leighton and Rao [15] used uniform multicommodity flow results to design the first polynomialtime (at most polylog $(n)$-times-optimal) approximation algorithms for wellknown NP-hard optimization problems such as graph partitioning, min-cut linear arrangement, crossing number, VLSI layout, and minimum feedback arc set.

Most work on analysing multicommodity flows has been directed towards developing algorithms (see for example [2, 3, 13, 14]). Theoretical studies require an underlying graph with some structure. Alongside the complete graph [4, the cube is one of the most natural examples, and has applications in the design of randomised routing algorithms for parallel computing (see Valiant [19]) and in random sampling of structures based on binary $d$-tuples.

\section{Definitions and preliminaries}

We recall some definitions and notation concerning flows. Let $G=G(V, E)$ be a graph. We denote the set of neighbours of a vertex $v$ by $\Gamma_{G}(v)$ and 
for simplicity we use $V \backslash v$ to mean $V \backslash\{v\}$. Suppose we have a network $\mathcal{N}$ consisting of an undirected graph $G$ together with a capacity $c_{e} \geq 0$ for each edge $e$. To define a (single commodity) flow in $\mathcal{N}$ we consider each undirected edge $e=u v$ as a pair of directed edges $\overrightarrow{u v}$ and $\overrightarrow{v u}$. Denote the set of all these directed edges by $\vec{E}$; and give each directed edge the same capacity as the original edge in $\mathcal{N}$.

Given a function $f: \vec{E} \rightarrow[0, \infty)$, the net outflow of $f$ at vertex $x$ is defined as

$$
f^{+}(x)=\sum_{y \in \Gamma_{G}(x)}(f(\overrightarrow{x y})-f(\overrightarrow{y x}))
$$

And the net inflow $f^{-}(x)$ is $-f^{+}(x)$.

For two disjoint, non-empty sets of vertices $S$ and $T$, we say that such a function is a proper $S T$ flow if it satisfies $f^{+}(x)=0$ for all $x \notin S \cup T$. A flow that is not proper is improper.

A proper $S T$ flow that also satisfies $f(\overrightarrow{x y}) \leq c(\overrightarrow{x y})$ for all $\overrightarrow{x y} \in \vec{E}$ is called a a feasible ST-flow. We also assume that for all $e=x y \in E$, either $f(\overrightarrow{x y})=0$ or $f(\overrightarrow{y x})=0$ and we write $f(e)=f(\overrightarrow{x y})+f(\overrightarrow{y x})$.

We choose the order of $S$ and $T$ such that $\sum_{x \in S} f^{+}(x) \geq 0$ and then the volume $\operatorname{vol}(f)$ is the magnitude of the flow given by $\operatorname{vol}(f)=\sum_{x \in S} f^{+}(x)=$ $\sum_{y \in T} f^{-}(y)$. In the special case when $S=\{s\}$ and $T=\{t\}$ we speak of an st-flow. See for example [1] for further discussion.

Let $\mathcal{V}$ be a non-empty set of unordered pairs of distinct vertices in the network $\mathcal{N}$. A multicommodity flow $F$ for $\mathcal{V}$ consists of an st-flow $f_{\text {st }}$ for each pair $\{s, t\}$ in $\mathcal{V}$ (which we arbitrarily order as st, the choice being immaterial as the underlying graph contains no directed edges). If each $f_{s t}$ has volume $\phi$ we say that $F$ is a uniform multicommodity flow of volume $\phi$. The total flow of $F$ in edge $e$ of $G$ is $\sum_{s t \in \mathcal{V}} f_{s t}(e)$; and $F$ is feasible if the total flow in each edge $e$ is at most its capacity $c_{e}$. The maximum uniform flow volume is the maximum value of $\phi$ such that there is a feasible uniform multicommodity flow of volume $\phi$. For a network $\mathcal{N}$ whose underlying graph is $Q^{d}$ and whose edge-capacities are independent, each distributed like a given random variable $C$ we say $\mathcal{N} \in \mathcal{G}\left(Q^{d}, C\right)$; and in the specific case of $C \sim$ $\operatorname{Ber}(p)$, the Bernoulli distribution with parameter $p$, we say $\mathcal{N} \in \mathcal{G}\left(Q^{d}, p\right)$.

We need two basic lemmas concerning tail probabilities.

Lemma 3. Given $1 / 2<p \leq 1$, there are constants $t>0, \tau>0$ such that

$$
\mathbb{P}[\operatorname{Bin}(d, p) \leq t d] \leq 2^{-(1+\tau) d} .
$$

Proof. Let $X \sim \operatorname{Bin}(d, p)$. For $t>0$ and $x \in(0,1)$, by Markov's inequality

$$
\left.(1-p+x p)^{d}=\mathbb{E}\left[x^{X}\right] \geq x^{t d} \mathbb{P}\left[x^{X} \geq x^{t d}\right]=x^{t d} \mathbb{P}[X \leq t d]\right] .
$$


And so

$$
\mathbb{P}[X \leq t d] \leq\left(x^{-t}(1-p+x p)\right)^{d} .
$$

We may pick $x \in(0,1)$ such that $1-p+x p<1 / 2$, and we then pick $t>0$ such that $x^{-t}(1-p+x p)<1 / 2$, finally we may choose $\tau>0$ so that $x^{-t}(1-p+x p)=2^{-(1+\tau)}$.

The following inequality is a form of Chernoff bound (see for example [5] Theorems A.1.4 and A.1.16).

Lemma 4. Let $X_{1}, \ldots, X_{n}$ be independent random variables with all $\left|X_{i}\right| \leq$ 1 , let $X=X_{1}+\cdots+X_{n}$, and let $Y=X-\mathbb{E} X$. Then for each $a \geq 0$,

$$
\mathbb{P}(Y \geq a) \leq e^{-a^{2} / 2 n} \quad \text { and } \quad \mathbb{P}(Y \leq-a) \leq e^{-a^{2} / 2 n} .
$$

\section{Upper bounds and convergence in expec- tation}

We need one deterministic lemma. Let $d(u, v)$ denote the number of edges in a shortest path between vertices $u$ and $v$.

Lemma 5. Let $\mathcal{N}$ be a network consisting of a graph $G$ together with a capacity $c_{e} \geq 0$ for each edge $e$; and let $\mathcal{V}$ be any non-empty collection of unordered pairs of distinct vertices of $G$ and let $\phi \geq 0$. If there is a feasible uniform multicommodity flow of volume $\phi$ between all pairs in $\mathcal{V}$ then

$$
\phi \sum_{\{u, v\} \in \mathcal{V}} d(u, v) \leq \sum_{e \in E(G)} c_{e} .
$$

Proof. Let $\{u, v\}$ be a pair in $\mathcal{V}$, suppose it is ordered as $u v$, and consider the flow $f_{u v}$ from $u$ to $v$. This flow can be decomposed as a sum of flows along paths from $u$ to $v$, together perhaps with some flows around cycles (see for example Ahuja, Magnanti and Orlin [1, page 80]). Since each of the paths has length at least $d(u, v)$, the total capacity used by $f_{u v}$ is at least $\phi \cdot d(u, v)$.

When $G$ is $Q^{d}$ and $\mathcal{V}$ is the collection of all $2^{d-1}$ antipodal pairs, then by the last lemma

$$
\phi \cdot d 2^{d-1} \leq \sum_{e \in E(G)} c_{e}=d 2^{d-1} c_{a v},
$$

where $c_{a v}$ is the average of the edge-capacities of $G$; and so

$$
\phi \leq c_{a v}
$$


Observe that for each vertex $u, 2^{-d} \sum_{v} d(u, v)=d / 2$. Thus if we take $\mathcal{V}$ as the collection of all pairs of distinct vertices then

$$
\sum_{\{u, v\} \in \mathcal{V}} d(u, v)=\frac{1}{2} \sum_{u, v} d(u, v)=\frac{1}{2} 2^{2 d} \frac{d}{2},
$$

and so as above

$$
\phi \cdot d 2^{2 d-2} \leq d 2^{d-1} c_{a v}
$$

that is

$$
\phi \cdot 2^{d-1} \leq c_{a v} .
$$

When we have random edge-capacities, $C_{a v}$ is the mean of $d 2^{d-1}$ iid (independent, identically distributed) random variables with finite mean $\mathbb{E}[C]$, and so by the weak law of large numbers, given $\epsilon>0, \mathbb{P}\left[C_{a v}>(1+\epsilon) \mathbb{E}[C]\right] \rightarrow 0$ as $d \rightarrow \infty$; and the upper bounds in Theorems 1 and 2 as in (1.1) follow from 3.1 and 3.2 .

Now consider expectations. Observe that always $\Phi_{\text {opp }} \geq 0$, and from (3.1) we have $\mathbb{E}\left[\Phi_{\text {opp }}\right] \leq \mathbb{E}[C]$. Thus once we have proved that $\Phi_{\text {opp }} \rightarrow \mathbb{E}[C]$ in probability it follows that $\mathbb{E}\left[\Phi_{\text {opp }}\right] \rightarrow \mathbb{E}[C]$. Similarly, from $(3.2)$ we see that $2^{d-1} \mathbb{E}\left[\Phi_{\text {all }}\right] \leq \mathbb{E}[C]$. Thus once we have proved that $2^{d-1} \Phi_{\text {all }} \rightarrow \mathbb{E}[C]$ in probability it follows that $2^{d-1} \mathbb{E}\left[\Phi_{\text {all }}\right] \rightarrow \mathbb{E}[C]$. Thus it remains to prove the lower bounds, as in (1.2), for convergence in probability.

\section{Antipodal flows: overview of lower bound proof}

We break the proof of the lower bound $\sqrt{1.2}$ in Theorem 1 down into $2^{d-1}$ separate parts, each of which concerns the flow of a single commodity between a pair of antipodal vertices. For each such pair we allocate a portion of the capacity of each edge according to scaling factors described in the next paragraphs. We find that, for each pair, there is only a very small probability that there fails to be a feasible flow of volume about $\mathbb{E}[C]$ in the restricted network (see Lemma 6). Then by taking the union bound, with high probability such flows exist simultaneously for all antipodal vertex pairs. When we superimpose these flows we need to sum the capacity used by all the separate flows; and we show that for every edge $e$ the total is at most $(1+o(1)) c_{e}$. Thus the theorem follows by rescaling flows and capacities.

In order to introduce the capacity scaling, let us first define the vertex and edge 'layers' from a given source vertex $u$ in $Q^{d}$. For $m=0,1, \ldots, d$ the vertex layer $V_{m}(u)$ consists of all the vertices at distance $m$ from $u$; and 
for $m=1, \ldots, d$ the edge layer $E_{m}(u)$ consists of all the edges between vertices in $V_{m-1}(u)$ and $V_{m}(u)$. We shall often take $\mathbf{0}$ (the $d$-vector of 0 's) as a representative vertex (note that the cube is vertex-transitive). Write $V_{m}$ for $V_{m}(\mathbf{0})$ and $E_{m}$ for $E_{m}(\mathbf{0})$, and note that $\left|V_{m}\right|=\left(\begin{array}{c}d \\ m\end{array}\right)$ and $\left|E_{m}\right|=m\left(\begin{array}{c}d \\ m\end{array}\right)$.

The capacity of each edge is divided amongst the $2^{d-1}$ sub-problems as follows. We fix a constant $1 / 2<\kappa<1$ and we define

$$
\ell=\ell(d)=\left\lfloor d^{\kappa}\right\rfloor .
$$

Here we think of the $\ell$ as local. The quantity $\kappa$ stays fixed throughout the paper. For a specific pair of antipodal vertices $u, \bar{u}$ the scaling is achieved in two stages. Firstly, for each $m$, each edge $e$ in layer $E_{m}(u)$ is given a capacity of $c_{e} /\left|E_{m}\right|$. We denote the network constructed so far by $\mathcal{N}(u)$. For the second stage we choose a (large) constant $M$ and scale up the capacities on the first and last $\ell+2$ edge layers, so the capacity of an edge in layer $E_{m}$ is

$$
\text { Scaled capacity }= \begin{cases}c_{e} /\left|E_{m}\right| & \text { if } \ell+3 \leq m \leq d-\ell-2 \\ M \cdot c_{e} /\left|E_{m}\right| & \text { if } m \leq \ell+2 \text { or } m \geq d-\ell-1 .\end{cases}
$$

The network with these scaled capacities is denoted by $\mathcal{N}^{M}(u)$, and is illustrated for the case $u=\mathbf{0}$ in Figure 1 .

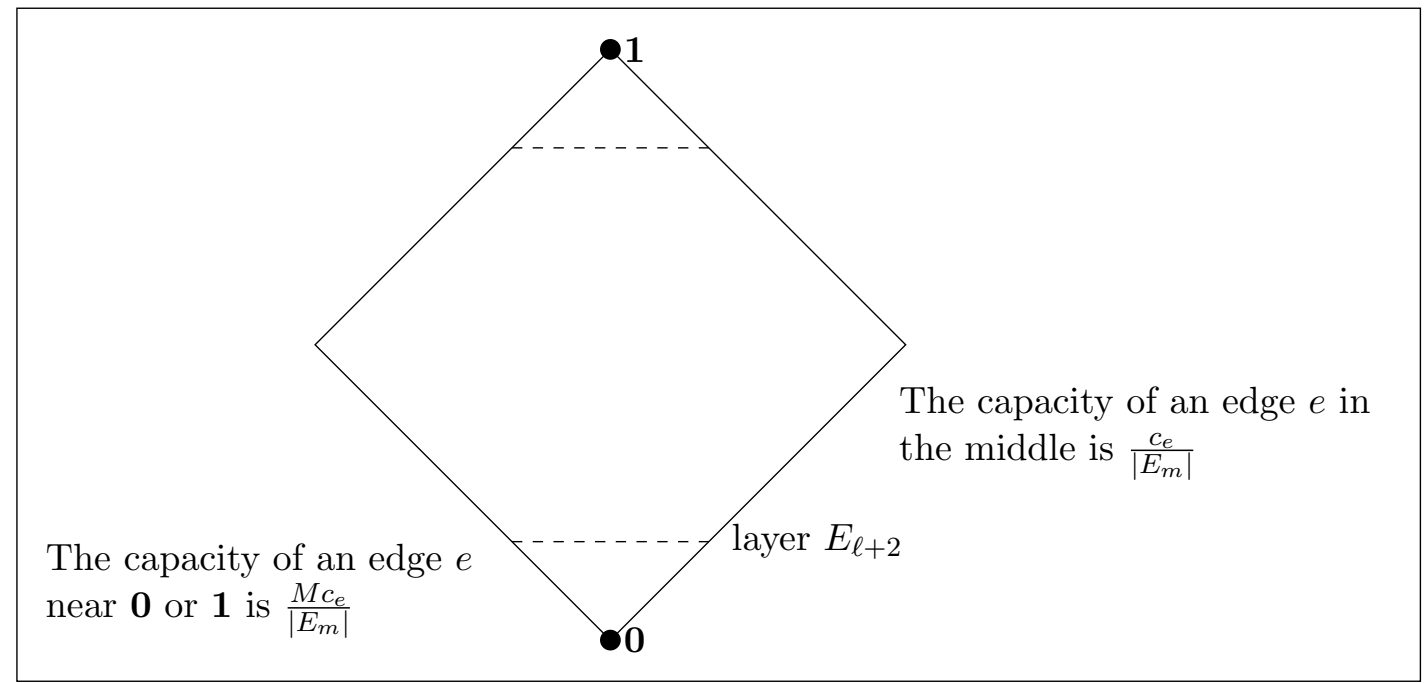

Figure 1: The scaled network $\mathcal{N}^{M}(\mathbf{0})$ illustrated as layers.

The first scaling provides sufficient capacity for the 'middle part' of the flow, where we are not near $u$ or $\bar{u}$. We shall see that the total of the 
capacities scaled by the first factor for a given edge $e$, summed over all the $2^{d-1}$ antipodal pairs, is exactly $c_{e}$.

The second scaling factor $M$ is introduced to enable flows to 'escape' from vertex $u$ and reach the boundary layer $V_{\ell}(u)$ (and similarly for $\bar{u}$ ). Due to local constraints, these flows may require capacity in an edge $e$ in the first $\ell+2$ edge-layers which is considerably greater than $c_{e} /\left|E_{m}\right|$, so the factor $M$ must be chosen sufficiently large. However, the impact of this 'profligate' use of capacity close to a source or sink turns out to be negligible, since $\kappa<1$ and thus $\ell=o(d)$. We shall see that we need the other bound on $\kappa$, namely that $\kappa>1 / 2$, in the proof of Lemma 15 (further details are given at the end of the proof of that lemma).

The following lemma is the main step in the proof of Theorem 1 .

Lemma 6. Given $\epsilon>0$ there are constant $M$ and $\rho>0$ such that the following holds. Let $\phi(u)$ be the maximum feasible flow volume between vertices $u$ and $\bar{u}$ in $\mathcal{N}^{M}(u)$. Then as $d \rightarrow \infty$,

$$
\mathbb{P}\left[\phi(u)<(1-\epsilon) \mathbb{E}[C] \text { for some } u \text { in } Q^{d}\right]=O\left(2^{-\rho d}\right) .
$$

In Sections 5 and 6 we establish preliminary results concerning flow close to the source and concerning the middle part of the flow, and in Section 7 we complete the proof of Lemma 6. It is helpful to work with 'balanced' and 'nearly balanced' flows. Given disjoint non-empty sets of vertices $S$ and $T$ in a network $\mathcal{N}$, and an $S T$ - flow $f$, we say that $f$ is balanced if the net outflow at each vertex in $S$ is $\operatorname{vol}(f) /|S|$ and the net inflow at each vertex in $T$ is $\operatorname{vol}(f) /|T|$. Given $\mu>0$, we say that $f$ is $\mu$-near-balanced if

$$
\sum_{v \in S}\left|f^{+}(v)-\frac{\operatorname{vol}(f)}{|S|}\right|+\sum_{v \in T}\left|f^{-}(v)-\frac{\operatorname{vol}(f)}{|T|}\right| \leq \mu \operatorname{vol}(f) .
$$

\section{Antipodal flows close to a source}

Our aim in this section is to show that with high probability (that is, with probability $\rightarrow 1$ as $d \rightarrow \infty$ ), for each vertex $u$ there is a balanced flow of volume $\mathbb{E}[C]$ from $u$ to $V_{\ell}(u)$ in $\mathcal{N}^{M}(u)$, when capacities are scaled by a suitable factor $M$.

We begin with the special case when capacities take only values 0 or 1 . The general case will follow easily. Given $0 \leq p \leq 1$ we let $Q_{p}=\left(Q^{d}\right)_{p}$ be the random subgraph of $Q^{d}$ where the edges appear independently with probability $p$. We also think of this as a random network based on $Q^{d}$ where the edge-capacity $C$ satisfies $\mathbb{P}(C=1)=p$ and $\mathbb{P}(C=0)=1-p$. 
For this case we show that (a) with high probability all vertices are suitably 'locally connected', (b) when this holds there must be a balanced flow of volume $\mathbb{E}[C]$ from $u$ to $V_{1}(u)$ when capacities are scaled by a suitable constant $M_{1}$, and (c) by scaling capacities by $M=7 M_{1}$ we may find a flow from $u$ to $V_{\ell}(u)$ as required. Parts (b) and (c) are deterministic.

The cube $Q^{d}$ can equivalently be defined by representing each vertex by a distinct subset of $[d]=\{1,2, \ldots, d\}$ with two vertices being adjacent when their symmetric difference is a singleton.

Each vertex in the cube $Q=Q^{d}$ has degree $d$. Let $u$ be a vertex of $Q$ and let $v \in \Gamma_{Q}(u)$. Then the edges in $Q$ between $\Gamma_{Q}(u) \backslash v$ and $\Gamma_{Q}(v) \backslash u$ form a perfect matching, of size $d-1$. Each edge is of the form $w_{1} w_{2}$, where $w_{1} \in V_{1}(u)$ and $w_{2} \in V_{2}(u)$. These edges $w_{1} w_{2}$, together with the edges $u w_{1}$ and $w_{2} v$ form $d-1$ internally vertex-disjoint $u v$-paths $u w_{1} w_{2} v$. See figure 2 , which illustrates the case $u=\emptyset$ (or $\mathbf{0}$ ) and $v=\{1\}$.

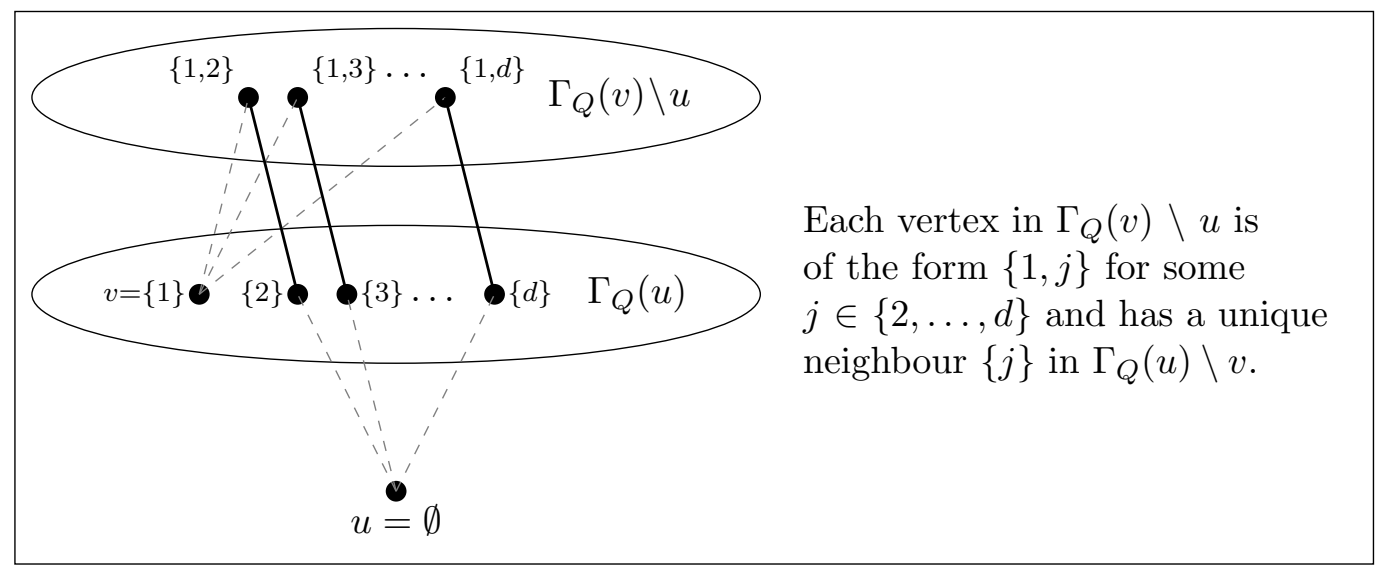

Figure 2: Matching between $\Gamma_{Q}(v) \backslash u$ and $\Gamma_{Q}(u) \backslash v$ with corresponding $u v$ paths.

We say a subgraph $R$ of $G$ is a spanning subgraph if $V(R)=V(G)$.

Definition 7. Let $0<\alpha<1$ be a constant. Let $R$ be a spanning subgraph of $Q=Q^{d}$. We say a vertex $u \in R$ is $\alpha$-locally-connected in $R$ if

1. its degree in $R$ is at least $\alpha d$, that is $\left|\Gamma_{R}(u)\right| \geq \alpha d$,

2. for each $v \in \Gamma_{Q}(u)$ there exists a matching in $R$ of size at least $\alpha d$ between $\Gamma_{Q}(u) \backslash v$ and $\Gamma_{Q}(v) \backslash u$,

3. For all but at most $\alpha^{-1}$ vertices $v \in \Gamma_{Q}(u)$ there exists a matching in $R$ of size at least $\alpha d$ between $\Gamma_{R}(u) \backslash v$ and $\Gamma_{R}(v) \backslash u$ (and so there are 
at least $\alpha$ internally vertex-disjoint uv paths of length 3 in the graph $R)$.

If all vertices in $R$ are $\alpha$-locally-connected in $R$ then we say that $R$ is $\alpha$ locally-connected. A vertex that is not $\alpha$-locally-connected is $\alpha$-poorly-connected. We denote the set of vertices that fail criterion $i$ above as $T_{i}^{\alpha}$ and the set of $\alpha$-poorly-connected vertices as $T^{\alpha}=T_{1}^{\alpha} \cup T_{2}^{\alpha} \cup T_{3}^{\alpha}$.

Once $d>\alpha^{-1}$ the first condition of the definition is implied by the third (so $T_{1}^{\alpha} \subseteq T_{3}^{\alpha}$ ). We now show that $Q_{p}$ is $\alpha$-locally-connected with high probability for sufficiently small $\alpha>0$.

Lemma 8. Given $1 / 2<p<1$, there are constants $\alpha>0, \rho>0$ such that $Q_{p}$ is $\alpha$-locally-connected with probability at least $1-2^{-\rho d}$.

Proof. Let $t>0, \tau>0$ be as in Lemma 3 and set $\alpha=\min t / 2,\left\lceil 34 / p^{4} t\right\rceil^{-1}$. Consider a fixed vertex $u$. We check whether $u$ is $\alpha$-locally-connected.

First condition. Since $\operatorname{deg}_{Q_{p}}(u) \sim \operatorname{Bin}(d, p)$,

$$
\mathbb{P}\left[\operatorname{deg}_{Q_{p}}(u) \leq t d\right] \leq 2^{-(1+\tau) d} .
$$

Second condition. For each $v \in \Gamma_{Q}(u)$ the edges in $Q$ between $\Gamma_{Q}(u) \backslash v$ and $\Gamma_{Q}(v) \backslash u$ form a matching of size $d-1$. The number, $X_{v}$, of these edges that are present in $Q_{p}$ has distribution $\operatorname{Bin}(d-1, p)$. Hence $\mathbb{P}\left[X_{v} \leq t(d-1)\right] \leq 2^{-(1+\tau)(d-1)}$. Thus for $d$ sufficiently large,

$$
\mathbb{P}\left[X_{v}<t d / 2 \text { for some } v \in \Gamma_{Q}(u)\right] \leq d 2^{-(1+\tau)(d-1)} \leq 2^{-(1+\tau / 2) d} .
$$

Third condition. We condition on $d_{Q_{p}}(u) \geq t$. Let $S \subseteq \Gamma_{Q}(u)$ with $|S| \geq t d$, and condition on $\Gamma_{Q_{p}}(u)=S$. Let $k \in \mathbb{N}$ and $S^{*} \subset V_{1}(u)$ with $\left|S^{*}\right|=k$. For each $v \in S^{*}$ let $Y_{v}$ be the number of paths in $Q_{p}$ of the form $u w_{1} w_{2} v$ where $w_{1} \in S \backslash S^{*}$ and $w_{2} \in V_{2}(u)$. Consider as an example (using the subset of $[d]$ notation introduced in Section 5 to denote our vertices) $u=\emptyset$, $v=\{1\}$ and $x \in S$ with $x=\{i\}$ : the only possible path is $\emptyset-\{i\}-\{1, i\}-\{1\}$, and the last two edges in this path are disjoint from paths from any other choice of $v$ or $x$. In general each edge in $E_{2}(u)$ is in at most one 3-edge path $u w_{1} w_{2} v$ where $w_{1} \in S \backslash S^{*}$ and $v \in S^{*}$. Also $\left|S \backslash S^{*}\right|>\frac{t d}{2}$ for large $d$ so each $Y_{v}$ is stochastically at least $\operatorname{Bin}\left(\left\lceil\frac{t d}{2}\right\rceil, p^{2}\right)$, and the random variables $Y_{v}$ for $v \in S^{*}$ are independent. (For two random variables $X$ and $Y$, we say $X$ is stochastically less than $Y$ if $\mathbb{P}[X \leq x] \geq \mathbb{P}[Y \leq x]$ for all possible $x$.)

We use the Chernoff Bounds as in Lemma 4, and set $s=p^{2}\left\lceil\frac{t d}{2}\right\rceil=$ $\mathbb{E}\left[\operatorname{Bin}\left(\left\lceil\frac{t d}{2}\right\rceil, p^{2}\right)\right]$ to get: 


$$
\mathbb{P}\left[Y_{v} \leq \frac{1}{4} p^{2} t d\right] \leq \mathbb{P}\left[\operatorname{Bin}\left(\left\lceil\frac{t d}{2}\right\rceil, p^{2}\right) \leq \frac{s}{2}\right] \leq e^{-\frac{1}{16} p^{4} t d}=: q .
$$

Let $A\left(S^{*}\right)$ be the event that $Y_{v} \leq \frac{1}{4} p^{2} t d$ for each vertex in $S^{*}$. Thus $\mathbb{P}\left[A\left(S^{*}\right)\right] \leq q^{k}$. There are $\left(\begin{array}{l}d \\ k\end{array}\right)$ possible choices of the set $S^{*}$ so, by the union bound, for sufficiently large $d$, the probability that $A\left(S^{*}\right)$ is true for one of these $k$-sets is at most

$$
\left(\begin{array}{l}
d \\
k
\end{array}\right) q^{k} \leq d^{k} e^{-\frac{1}{16} p^{4} t k d} \leq e^{-\frac{1}{17} p^{4} t k d}
$$

So we choose $k=\left\lceil 34 / p^{4} t\right\rceil$ and with failure probability at most $e^{-2 d}$ there is no subset of $V_{1}(u)$ of size $k$ with every vertex having the property $Y_{v} \leq \frac{1}{4} p^{2} t d$. Now we can remove the conditioning that $\Gamma_{Q_{p}}(u)=S$, and we see that, conditional on $d_{Q_{p}}(u) \geq t d$, the probability that $A\left(S^{*}\right)$ holds for some $k$ subset of $V_{1}(u)$ is at most $e^{-2 d}$.

Now $\mathbb{P}\left[u \in T^{\alpha}\right] \leq \mathbb{P}\left[u \in T_{1}^{\alpha}\right]+\mathbb{P}\left[u \in T_{2}^{\alpha}\right]+\mathbb{P}\left[u \in T_{3}^{\alpha} \mid u \notin T_{1}^{\alpha}\right]$. Using equation (5.1) $\mathbb{P}\left[u \in T_{1}^{\alpha}\right] \leq 2^{-(1+\tau) d}$, and for large $d$, from equation (5.2) $\mathbb{P}\left[u \in T_{2}^{\alpha}\right] \leq 2^{-(1+\tau / 2) d}$ and from the previous section $\mathbb{P}\left[u \in T_{3}^{\alpha} \mid u \notin T_{1}^{\alpha}\right] \leq$ $e^{-2 d}$. Thus, for large $d$, the probability that $u$ is not $\alpha$-locally-connected is at most $2^{-(1+\rho) d}$ where $\rho=\tau / 3$; and by the union bound, $Q_{p}$ is $\alpha$-locallyconnected with probability at least $1-2^{-\rho d}$.

We denote by $B_{r}^{M}(u)$ the ball of radius $r$ centred on vertex $u$ with the capacities scaled as in $\mathcal{N}^{M}(u)$. Thus $B_{r}^{M}(u)$ is $\mathcal{N}^{M}(u)$ restricted to the vertex layers up to $V_{r}(u)$. We say $\mathcal{N}$ has the local escape property with parameter $M$ if, for each vertex $u$, we can route a balanced feasible flow of volume 1 between $u$ and $\Gamma_{Q}(u)$ using only paths in $B_{3}^{M}(u)$.

We now prove two deterministic lemmas.

Lemma 9. Given $0<\alpha<1$ there exists a constant $M_{1}=M_{1}(\alpha)>0$ such that the following holds. Let the spanning subgraph $R$ of $Q$ be $\alpha$-locallyconnected, and let $\mathcal{N}$ be the network formed by giving capacity 1 to each edge of $R$. Then $\mathcal{N}$ has the local escape property with parameter $M_{1}$.

Proof. Consider vertex $u$. Let $S_{1}=S_{1}(u)=\Gamma_{R}(u)$, let $S_{3}=S_{3}(u)$ be the set of vertices in $\Gamma_{Q}(u) \backslash S_{1}$ for which the number of paths of length 3 in $R$ to $u$ is greater than $\alpha d$, and denote the remainder $\left(\Gamma_{Q}(u) \backslash\left(S_{1} \cup S_{3}\right)\right)$ by $S^{*}=S^{*}(u)$. Since $R$ is $\alpha$-locally-connected we have $\left|S_{1}\right| \geq \alpha d$ and $\left|S^{*}\right| \leq\left\lfloor\alpha^{-1}\right\rfloor=: N$. We now route flows as follows.

For $v \in S_{1}$ we route a flow of $d^{-1}$ along edge $u v$. For $v \in S_{3}$ we route a flow of $d^{-1}$ split evenly between $\lceil\alpha d\rceil$ of the paths of length 3 from $u$ to $v$ (chosen 
arbitrarily). The total of these flows is at most $(\alpha d)^{-1} d^{-1}<\alpha^{-1}\left|E_{2}\right|^{-1}$ in any edge in $E_{2}=E_{2}(u)$ and at most $\left(1+\alpha^{-1}\right) d^{-1}$ in any edge in layer $E_{1}=E_{1}(u)$. Thus we can route flows of volume $d^{-1}$ to all vertices in $S_{1} \cup S_{3}$ in $B_{u}^{M}(2)$ for $M_{0}=1+\alpha^{-1}$.

For $v \in S^{*}$ we know there is a matching in $R$ of size at least $\alpha d$ between $\Gamma_{Q}(u) \backslash v$ and $\Gamma_{Q}(v) \backslash u$, so there is a matching in $R$ of size at least $\alpha d-$ $\left|S^{*}(u)\right|-\left|S^{*}(v)\right| \geq \alpha d-2 N$ between $S_{1}(u) \cup S_{3}(u)$ and $S_{1}(v) \cup S_{3}(v)$. For large enough $d$, we may pick a matching of size $\lceil\alpha d / 2\rceil$, say $v_{1} w_{1}, v_{2} w_{2}, \ldots$ where $v_{i} \in S_{1}(v) \cup S_{3}(v)$ and $w_{i} \in S_{1}(u) \cup S_{3}(u)$. We route a flow of volume $\lceil\alpha d / 2\rceil^{-1} d^{-1}$ along each of these edges. We route the flows from $u$ to the $v_{i}$ by scaling the flows already found above by a factor $1+\lceil\alpha d / 2\rceil^{-1}$ and from the $w_{i}$ to $v$ using the routes already found by the same method and using the same scaling factor. There are at most $N$ vertices in $S^{*}$ so the volume from all the flows from $u$ to $S^{*}$ is at most $N M_{0}\lceil\alpha d / 2\rceil^{-1} d^{-2} \leq \frac{M_{0} N}{\alpha}\left|E_{3}\right|^{-1}$ in any edge in $E_{3}=E_{3}(v)$ and $N M_{0}\lceil\alpha d / 2\rceil^{-1} d^{-1} \leq \frac{M_{0} N}{\alpha}\left|E_{2}\right|^{-1}$ in any edge in layer $E_{2}$ and $N\lceil\alpha d\rceil^{-1} d^{-1} \leq \frac{M_{0} N}{\alpha}\left|E_{1}\right|^{-1}$ in any edge in $E_{1}$. Thus we can route flows of volume 1 to all vertices in $\Gamma_{Q}(u)$ in $B_{3}^{M_{1}}(u)$ for $M_{1}=\frac{M_{0} N}{\alpha}$.

Lemma 10. Let $R$ be a spanning subgraph of $Q$ and let $\mathcal{N}$ be the network formed by giving a capacity of 1 to each edge of $R$. Assume that $\mathcal{N}$ has the local escape property with parameter $M_{1}$. Then, for $d$ sufficiently large, for each $u \in V(Q)$ there exists a balanced flow of volume 1 between $u$ and $V_{\ell}(u)$ in $B_{u}^{M}(\ell+2)$, where $M=7 M_{1}$.

Proof. Fix $u \in V(Q)$. Let $1 \leq m \leq \ell$ and let $v \in V_{m-1}(u)$. By our assumption on $\mathcal{N}$, there is a balanced flow of volume $\frac{d}{d-m+1}\left|V_{m-1}\right|^{-1}$ from $v$ to all of its neighbours in $Q$ in the ball $B_{3}^{M_{2}}(v)$ where $M_{2}=\frac{d}{d-m+1}\left|V_{m-1}\right|^{-1} M_{1}$. We decompose this flow into flows along paths of length at most 7 and only consider those flows to neighbours of $v$ in $V_{m}$. This results in separate flows of volume $\frac{1}{d-m+1}\left|V_{m-1}\right|^{-1}$ from $v$ to each of its neighbours in $V_{m}$. Repeating this process for every vertex in $V_{m-1}$ gives us a flow to every vertex in $V_{m}$ of volume $\frac{m}{d-m+1}\left|V_{m-1}\right|^{-1}=\left|V_{m}\right|^{-1}$. Thus a balanced flow of volume 1 exists between $V_{m-1}$ and $V_{m}$ if each edge has the capacity given by the addition of the capacities of that edge in the balls $B_{3}^{M_{1}}(v)$ for all $v \in V_{m-1}$ scaled by the factor $\frac{d}{d-m+1}\left|V_{m-1}\right|^{-1}$. We repeat this process for all vertex layers 1 to $\ell$ to get a balanced flow of volume 1 from $u$ to $V_{\ell}(u)$.

To calculate an upper bound to the total capacity required in edge $e=x y$ $\left(x \in V_{m-1}, y \in V_{m}\right)$ we need to consider all of the balls centred on vertices a distance at most 2 from $x$ or $y$. The capacity required in $e$ for one of these balls is the product of the scaling factor applied to the ball (the factor $\frac{d}{d-m+1}\left|V_{m-1}\right|^{-1} M_{1}$ for a ball centred on a vertex in $\left.V_{m-1}\right)$ and the factor 
applied to edge $e$ in the ball. $\left(\left(\begin{array}{l}d \\ k\end{array}\right)^{-1}\right.$ if $e$ is in edge-layer $k$ in the ball.) The vertices within a distance 2 of $x$ can be partitioned into 6 sets.

$$
\begin{aligned}
& S_{1}=\{x\},\left|S_{1}\right|=1 \\
& S_{2}=\left\{z \in V_{m-2}: d_{Q}(x z)=1\right\}, \text { where }\left|S_{2}\right|=m-1 \\
& S_{3}=\left\{z \in V_{m}: d_{Q}(x z)=1\right\}, \text { where }\left|S_{3}\right|=d-m+1 \\
& S_{4}=\left\{z \in V_{m-3}: d_{Q}(x z)=2\right\}, \text { where }\left|S_{4}\right|=\left(\begin{array}{c}
m-1 \\
2
\end{array}\right) \\
& S_{5}=\left\{z \in V_{m-1}: d_{Q}(x z)=2\right\}, \text { where }\left|S_{5}\right|=(m-1)(d-m+1) \\
& S_{2}=\left\{z \in V_{m+1}: d_{Q}(x z)=2\right\}, \text { where }\left|S_{6}\right|=\left(\begin{array}{c}
d-m+1 \\
2
\end{array}\right)
\end{aligned}
$$

We denote by $C_{i}$ the total capacity required in edge $e$ for the balls centred on vertices in set $S_{i}$ and

$$
\begin{aligned}
& C_{1}=\left|S_{1}\right| \frac{d}{d-m+1}\left|V_{m-1}\right|^{-1} \frac{M_{1}}{d}=M_{1}\left(\left|V_{m-1}\right| d\right)^{-1}(1+O(m / d)) \\
& C_{2}=\left|S_{2}\right| \frac{d}{d-m+2}\left|V_{m-2}\right|^{-1} M_{1}\left(\begin{array}{l}
d \\
2
\end{array}\right)^{-1}=2 M_{1}\left(\left|V_{m-1}\right| d\right)^{-1}(1+O(m / d)) \\
& C_{3}=\left|S_{3}\right| \frac{d}{d-m}\left|V_{m}\right|^{-1} M_{1}\left(\begin{array}{l}
d \\
2
\end{array}\right)^{-1}=O\left(\frac{m}{d} M_{1}\left(\left|V_{m-1}\right| d\right)^{-1}\right) \\
& C_{4}=\left|S_{4}\right| \frac{d}{d-m+3}\left|V_{m-3}\right|^{-1} M_{1}\left(\begin{array}{l}
d \\
3
\end{array}\right)^{-1}=3 M_{1}\left(\left|V_{m-1}\right| d\right)^{-1}(1+O(m / d)) \\
& C_{5}=\left|S_{5}\right| \frac{d}{d-m+1}\left|V_{m-1}\right|^{-1} M_{1}\left(\begin{array}{l}
d \\
3
\end{array}\right)^{-1}=O\left(\frac{m}{d} M_{1}\left(\left|V_{m-1}\right| d\right)^{-1}\right) \\
& C_{6}=\left|S_{6}\right| \frac{d}{d-m-1}\left|V_{m+1}\right|^{-1} M_{1}\left(\begin{array}{l}
d \\
3
\end{array}\right)^{-1}=O\left(\frac{m}{d} M_{1}\left(\left|V_{m-1}\right| d\right)^{-1}\right)
\end{aligned}
$$

And the sum of these is $\sum C_{i}=6 M_{1}\left(\left|V_{m-1}\right| d\right)^{-1}(1+O(m / d))$. We do a similar exercise for balls centred on vertices a distance at most 2 from $y$ and get the total capacity required for all these balls is $6 M_{1}\left(\left|V_{m}\right| d\right)^{-1}(1+O(m / d))$. We note that $\left|V_{m}\right|^{-1}=O\left(m / d\left|V_{m-1}\right|^{-1}\right)$, so the total capacity required in edge $e$ from all balls centred on vertices a distance at most 2 from either $x$ or $y$ is $6 M_{1}\left(\left|V_{m-1}\right| d\right)^{-1}(1+O(m / d))$. Putting $M=7 M_{1}$ gives the result.

Putting Lemmas 8, 9 and 10 together, we obtain the following lemma.

Lemma 11. Given $1 / 2<p<1$, there exist constants $M>0$, and $\rho>0$ such that for $d$ sufficiently large, with failure probability at most $2^{-\rho d}$, for all 
vertices $u$ in $Q_{p}$ there exists a balanced flow of volume 1 in the ball $B_{\ell+2}^{M}(u)$ from $u$ to $V_{\ell}(u)$.

We can now prove the main result of this section, Lemma 12. In Lemma 11 we proved the result in the special case when $C \sim \operatorname{Ber}(p)$, the Bernoulli distribution with parameter $p$.

Lemma 12. For a random variable $C$ with $\mathbb{P}[C>0]>1 / 2$ there exist constants $M>0$ and $\rho>0$ such that the following holds with failure probability at most $2^{-\rho d}$. For $\mathcal{N} \in \mathcal{G}(Q, C)$, for all vertices $u$ there exists a balanced flow of volume $\mathbb{E}[C]$ in the ball $B_{\ell+2}^{M}(u)$ from $u$ to $V_{\ell}(u)$.

Proof. Since $\mathbb{P}[C>0]>1 / 2$, there is $c^{*}>0$ such that $\mathbb{P}\left[C \geq c^{*}\right]>1 / 2$. We denote $\mathbb{P}\left[C \geq c^{*}\right]$ by $p^{*}$. We now consider the network $\mathcal{N}^{*}$ which is the network $\mathcal{N}$ with edge-capacities reduced as follows. For an edge $e$ with $C_{\mathcal{N}}(e)<c^{*}$ we put $C_{\mathcal{N}^{*}}(e)=0$ and for an edge $e$ with $C_{\mathcal{N}}(e) \geq c^{*}$ we put $C_{\mathcal{N}^{*}}(e)=c^{*}$. Thus the edge-capacities of $\mathcal{N}^{*}$ have distribution $c^{*} \operatorname{Ber}\left(p^{*}\right)$ and the capacity of each edge in $G^{*}$ is at most its capacity in $G$. From Lemma 11 we know that there exists $M^{\prime}$ and $\rho>0$ such that with failure probability at most $2^{-\rho d}$, for all vertices $u$, there exists a balanced flow of volume 1 in the ball $B_{\ell+2}^{M^{\prime}}(u)$ from $u$ to $V_{\ell}(u)$. Putting $M=\frac{M^{\prime} \mathbb{E}[C]}{c^{*}}$ we get the result.

\section{Antipodal flows in the middle part of a flow}

Our aim in this section is to show that, with failure probability $O\left(e^{-d^{2}}\right)$, there is a $d^{-2}$-near-balanced flow of volume $(1+\epsilon)^{-1} \mathbb{E}[C]$ from $V_{\ell}(\mathbf{0})$ to $V_{\ell}(\mathbf{1})$ in our scaled network $\mathcal{N}(\mathbf{0})$. (Recall the definitions of $\mathcal{N}(\mathbf{0})$ and near-balanced flows from Section 4.)

Once again we do most of the work with the Bernoulli distribution $C \sim$ $\operatorname{Ber}(p)$ for $0<p<1$. (Note that we do not now require $p>1 / 2$.)

The proof proceeds from layer to layer by showing (in Lemma 15) that, with high probability, a $d^{-3}$-near-balanced flow can be routed across edge layer $E_{m}$ which forms a bipartite network $\mathcal{B}_{m}$. Lemma 17 puts together the flows across the layers to find the desired flow from $V_{\ell}(\mathbf{0})$ to $V_{\ell}(\mathbf{1})$. In preparation we need the following two lemmas.

In the proof of Lemma 15 we may have, for each $m$, small subsets of $V_{m}$ which do not have some property we would like. The following lemma bounds the impact that these 'bad' vertices can have.

Lemma 13. Let $\ell \leq m \leq d-\ell$; let $\left\{X_{i}: 1 \leq i \leq\left(\begin{array}{c}d \\ m\end{array}\right)\right\}$ be a family of independent random variables taking values 0 or 1 where $\mathbb{P}\left[X_{i}=1\right] \leq d^{-100}$ 
for all $i$, and let $S=\sum_{i} X_{i}$. Then, for large $d$,

$$
\mathbb{P}\left[S \geq\left(\begin{array}{c}
d \\
m
\end{array}\right) d^{-99}\right] \leq e^{-d^{2}}
$$

Proof. By Lemma 4, we have

$$
\mathbb{P}\left[S \geq\left(\begin{array}{c}
d \\
m
\end{array}\right) d^{-99}\right] \leq \mathbb{P}\left[S-\mathbb{E}[S] \geq \frac{1}{2}\left(\begin{array}{c}
d \\
m
\end{array}\right) d^{-99}\right] \leq e^{-\frac{1}{8}\left(\begin{array}{c}
d \\
m
\end{array}\right) d^{-198}}
$$

But $\left(\begin{array}{c}d \\ m\end{array}\right) \geq\left(\begin{array}{l}d \\ \ell\end{array}\right) \geq\left(\frac{d}{\ell}\right)^{\ell}=d^{(1-\kappa) d^{\kappa}+O(1)} \geq 8 d^{200}$, for large $d$.

The proof of Lemma 15 considers the bipartite network of edges across layer $m$ as the network $\mathcal{B}^{*}$ formed by the superposition of two independent networks: $\mathcal{B}^{\prime}$ in which edges are present with probability $p^{\prime}$, with $p^{\prime}$ close to but less than $p$ and $\mathcal{B}_{\delta}$ in which edges are present with small probability $\delta$. The proof of Lemma 15 looks at the flow imbalances that occur at each vertex in the $\mathcal{B}^{\prime}$ network if a uniform flow reaches one vertex class $V_{m-1}$, a uniform flow leaves the other vertex class $V_{m}$, and the full capacity of every edge in $\mathcal{B}^{\prime}$ connecting the two classes is used. We show that at all except a small number of vertices these imbalances are small. We then use the network $\mathcal{B}_{\delta}$ to smooth these imbalances so they are very small. We need the following technical lemma to quantify this 'smoothing'.

Lemma 14. Fix $\delta, \lambda$ with $1 / 2<\lambda<\kappa<1$ and $0<\delta<1$. Fix $m$ such that $d^{\kappa}<m<d / 2$ and let $\psi_{\max }=2 d^{\lambda}\left|E_{m}\right|^{-1}$. Let $G_{\delta}$ be the bipartite graph with vertex classes $V_{m-1}$ and $V_{m}$, in which edges of $Q^{d}$ are present independently with probability $\delta$; and let $\mathcal{B}_{\delta}$ be the network formed by giving each edge of $G_{\delta}$ capacity $d^{(\lambda-\kappa) / 2}\left|E_{m}\right|^{-1}$. Let $V=V_{m} \cup V_{m-1}$. Let $\{\psi(x): x \in V\}$ be a family of random variables such that the following hold:

- the random variables $(\psi(x))_{x \in V}$ are independent of the edge-capacities,

- the random variables $(\psi(x))_{x \in V_{m-1}}$ are independent,

- the random variables $(\psi(x))_{x \in V_{m}}$ are independent,

- for each $x \in V,|\psi(x)| \leq \psi_{\max }$,

- $\mathbb{E}[\psi(x)]=0$ for all $x$.

Then, with failure probability $O\left(e^{-d^{2}}\right)$, there is a function $\theta: V \rightarrow \mathbb{R}$ and a feasible flow in $\mathcal{B}_{\delta}$ such that the net inflow at each vertex is $\psi(x)+\theta(x)$ and $\sum_{x \in V}|\theta(x)| \leq d^{-4}$. 
Proof. We denote by $T^{s}$ ( $s$ for small degree) the set of vertices in $G_{\delta}$ with degree less than half their expected values $(\delta m / 2$ or $\delta(d-m+1) / 2$ for vertices in $V_{m}$ and $V_{m-1}$ respectively). We start by showing that there are very few vertices in $G_{\delta}$ for which there is a vertex in $T^{s}$ within a distance 40 in $Q$. Let

$$
\begin{aligned}
T_{m}^{2} & =\left\{u \in V_{m}: \exists v \in T^{s} \text { with } d_{Q}(u, v) \leq 40\right\}, \\
T_{m-1}^{2} & =\left\{u \in V_{m-1}: \exists v \in T^{s} \text { with } d_{Q}(u, v) \leq 40\right\}, \\
T^{2} & =T_{m-1}^{2} \cup T_{m}^{2} .
\end{aligned}
$$

The events $d_{G_{\delta}}(v)<\delta m / 2$ for $v \in V_{m}$ are independent and, by Lemma 4. $\mathbb{P}\left[d_{G_{\delta}}(v)<\delta m / 2\right] \leq e^{-\delta^{2} m^{2} / 8 d} \leq e^{-\delta^{2} d^{2 \kappa-1} / 8} \leq d^{-100}$ for large $d$. Then the distribution of the number of vertices with degree less than half their expected value is stochastically less than $\operatorname{Bin}\left(\left|V_{m}\right|, d^{-100}\right)$, and so by Lemma 13. the number is less than $\left|V_{m}\right| d^{-99}$ with failure probability $O\left(e^{-d^{2}}\right)$. Each of these vertices is within a distance 40 of at most $d^{40}$ other vertices so $\mathbb{P}\left[\left|T_{m}^{2}\right| \geq\right.$ $\left.\left|V_{m}\right| d^{-59}\right]=O\left(e^{-d^{2}}\right)$. A similar argument shows $\mathbb{P}\left[\left|T_{m-1}^{2}\right| \geq\left|V_{m-1}\right| d^{-59}\right]=$ $O\left(e^{-d^{2}}\right)$.

Let $\mathcal{B}$ be any bipartite network with vertex classes $V_{m-1}$ and $V_{m}$ and edgecapacities $d^{(\lambda-\kappa) / 2}\left|E_{m}\right|^{-1}$ and also with the properties that $\left|T_{m}^{2}\right| \leq\left|V_{m}\right| d^{-59}$ and $\left|T_{m-1}^{2}\right| \leq\left|V_{m-1}\right| d^{-59}$. We condition on the network $\mathcal{B}_{\delta}$ being the network $\mathcal{B}$.

For each vertex $x \in V \backslash T^{2}$ we use the open edges in $\mathcal{B}$ to carry a flow of volume $|\psi(x)|$ to or from (depending on whether $\psi(x)>0$ or $\psi(x)<0$ ) the vertices $y$ where $d_{Q}(y, x)=d_{\mathcal{B}}(y, x)=40$. If $\psi(x)>0$ the flows are constructed by pushing a flow of magnitude $\psi(x)$ equally to all the neighbours of $x$ in $\mathcal{B}$ and for each of those vertices then splitting the flow equally between its neighbours in $\mathcal{B}$ a distance one further away from $x$ in both $Q$ and $\mathcal{B}$ and so on. If $\psi(x)<0$ a similar flow of volume $-\psi(x)$ is constructed in the opposite direction. We shall see that, since $x \in V \backslash T^{2}$, there are always many neighbours one further away from $x$ in both $Q$ and $\mathcal{B}$.

We denote by $f_{x}(z)$ the amount of flow that reaches (or comes from) a destination vertex $z$ from a source (or sink) $x$. $\left(f_{x}(z)\right.$ has the same sign as $\psi(x))$. We denote by $X(z)$ the set of vertices that might send a flow to a destination $z$ (or receive from $z$ if $\psi(x)<0$ ) and by $F(z)=\sum_{x \in X(z)} f_{x}(z)$, the resultant flow at $z$.

Two vertices $x, z$ in $V_{m}$ (or equally $V_{m-1}$ ) are separated by a distance 40 (in $Q$ ) if 20 of the elements of (the set representing) $x$ are replaced by 20 elements of $[d] \backslash x$ to obtain (the set representing) $z$. For each $x \in V_{m}$ there are therefore $\left(\begin{array}{c}m \\ 20\end{array}\right)\left(\begin{array}{c}d-m \\ 20\end{array}\right)$ such vertices, and for each $x \in V_{m-1}$ there are $\left(\begin{array}{c}m-1 \\ 20\end{array}\right)\left(\begin{array}{c}d-m+1 \\ 20\end{array}\right)$ such vertices. 
The magnitude $\left|f_{x}(z)\right|$ depends on the size of the initial imbalance $|\psi(x)|$, the number of paths from $x$ to $z$ that exist in $\mathcal{B}$ and the 'onward vertex degree' (i.e. the number of edges in $\mathcal{B}$ leaving a vertex on the path to a vertex further away in $Q$ and $\mathcal{B}$ from $x$ ) of each vertex on these paths. For a given $x$ and $z$ there are $(20 \text { ! })^{2}$ possible paths from $x$ to $z$ corresponding to the different possible orders of removing and adding elements from $x$ to reach $z$. For a particular path $\mathcal{P}$ we define $\nu(\mathcal{P})=1$ if all edges in $\mathcal{P}$ are open and $\nu(\mathcal{P})=0$ otherwise and we denote by $c(\mathcal{P})$ the product of the onward degrees along $\mathcal{P}$. Then $f_{x}(z)$ can be expressed as

$$
f_{x}(z)=\psi(x) \sum_{\mathcal{P}} \frac{\nu(\mathcal{P})}{c(\mathcal{P})},
$$

where the sum is taken over all $(20 !)^{2}$ paths of length 40 from $x$ to $z$. We make two observations about this expression. Firstly, the sum $\sum_{\mathcal{P}} \frac{\nu(\mathcal{P})}{c(\mathcal{P})}$ depends only on which edges in $\mathcal{B}$ are open and is fixed as we are conditioning on $\mathcal{B}$. For a given $z$ the variables $\left\{f_{x}(z): x \in V_{m}\right\}$ are therefore independent (though not, in general, with the same distribution). Secondly, each vertex in the path has degree at least half its expected degree (either $\delta m / 2$ or $\delta(d-m+1) / 2)$. Therefore,

$$
c(\mathcal{P}) \geq \prod_{i=1}^{20}\left(\frac{m \delta}{2}-i\right)\left(\frac{(d-m+1) \delta}{2}-i\right),
$$

and so

$$
m^{20}(d-m+1)^{20} \geq c(\mathcal{P}) \geq m^{20}(d-m+1)^{20}(\delta / 3)^{40} .
$$

Since $d / 2 \leq d-m+1 \leq d$,

$$
\left|f_{x}(z)\right| \leq \frac{c \psi_{\max }}{(d m)^{20}}
$$

where $c=(20 !)^{2}(3 / \delta)^{40} 2^{20}$.

The expressions within the sum $F(z)=\sum_{x \in X(z)} f_{x}(z)$ are independent, bounded and have zero expectation, so by the Chernoff bounds (Lemma 4),

$$
\mathbb{P}\left[|F(z)|>a \frac{c \psi_{\max }}{(m d)^{20}}\right]<2 e^{-a^{2} / 2|X(z)|} .
$$

We note $|X(z)| \leq\left(\begin{array}{c}d \\ 20\end{array}\right)^{2} \leq d^{40} / 2$ and put $a=d^{20+4 / 3}$ to get $\frac{a^{2}}{2|X(z)|} \geq d^{8 / 3}$. Since $\psi_{\max }=2 d^{\lambda}\left|E_{m}\right|^{-1}$ and $(m d)^{20} \geq d^{(1+\kappa) 20}$, we get

$$
\mathbb{P}\left[|F(z)|>\left(\left|E_{m}\right| d^{5}\right)^{-1}\right]=O\left(e^{-2 d^{2}}\right) .
$$


This result holds for each $z \in V$ and so

$$
\mathbb{P}\left[|F(z)|>\left(\left|E_{m}\right| d^{5}\right)^{-1} \text { for some } z \in V\right]=O\left(e^{-d^{2}}\right) .
$$

After this process we have the original imbalances $\psi(z)$ at vertices in $T^{2}$ which were not 'smoothed', and the imbalances $F(z)$ arising from the smoothing process. We now introduce an equal and opposite flow $\theta(z)$ into every vertex in $\mathcal{B}$ to make the overall flow feasible. For $z \in T^{2}$, we set $\theta(z)=-\psi(z)-F(z)$ and for $z \in V \backslash T^{2}$, we set $\theta(z)=-F(z)$. So

$$
\sum_{z \in V}|\theta(z)| \leq \sum_{z \in V}|F(z)|+\sum_{z \in T^{2}}|\psi(z)|
$$

For $z \in T_{m}^{2}$, we have $|\psi(z)| \leq \psi_{\max } \leq\left|V_{m}\right|^{-1}$ for large $d$; and similarly, for $z \in T_{m-1}^{2}$, we have $|\psi(z)| \leq\left|V_{m-1}\right|^{-1}$. So then

$$
\sum_{z \in T^{2}}|\psi(z)| \leq\left|T_{m}^{2}\right|\left|V_{m}\right|^{-1}+\left|T_{m-1}^{2}\right|\left|V_{m-1}\right|^{-1} \leq 2 d^{-59}
$$

We also have $\sum_{z \in V_{m}}|F(z)| \leq\left|V_{m}\right|\left|E_{m}\right|^{-1} d^{-5} \leq d^{-5}$ with failure probability $O\left(e^{-d^{2}}\right)$ and similarly $\sum_{z \in V_{m-1}}|F(z)| \leq\left|V_{m-1}\right|\left|E_{m}\right|^{-1} d^{-5} \leq d^{-5}$ with failure probability $O\left(e^{-d^{2}}\right)$. So with failure probability $O\left(e^{-d^{2}}\right)$,

$$
\sum_{x \in V}|\theta(x)| \leq d^{-4}
$$

We must check that these flows do not exceed the capacities of $\mathcal{B}$. Consider edge $e=u v$ where $u \in V_{m-1}$ and $v \in V_{m}$. We denote by $F(e)=$ $\sum_{\mathcal{P}} f_{x}(z)$ the total flow in edge $e$ of all the $f_{x}(z)$ flows for all possible $x$ and for all possible $z$ where $e$ is in a path in $Q[V]$ of length 40 from $x$ to $z$. The total possible number of paths of length 40 is $\left|V_{m}\right|\left(\begin{array}{c}m \\ 20\end{array}\right)\left(\begin{array}{c}d-m \\ 20\end{array}\right)+\left|V_{m-1}\right|\left(\begin{array}{c}m-1 \\ 20\end{array}\right)\left(\begin{array}{c}d-m+1 \\ 20\end{array}\right)$ and so, by the symmetry of the cube, each edge is in at most 40 times this number divided by the total number of edges (i.e. $\left|E_{m}\right|=\left|V_{m}\right| m=$ $\left.\left|V_{m-1}\right|(d-m+1)\right)$. So each edge is in

$$
\frac{40}{m}\left(\begin{array}{c}
m \\
20
\end{array}\right)\left(\begin{array}{c}
d-m \\
20
\end{array}\right)+\frac{40}{d-m+1}\left(\begin{array}{c}
m-1 \\
20
\end{array}\right)\left(\begin{array}{c}
d-m+1 \\
20
\end{array}\right)=O\left(\frac{(m d)^{20}}{m}\right)
$$

paths. So $|F(e)|$ is at most this number of paths times the maximum value of any individual $\left|f_{x}(z)\right|$ i.e.

$$
|F(e)|=O\left(\frac{(m d)^{20}}{m} \frac{\psi_{\max }}{(m d)^{20}}\right)=O\left(\frac{\psi_{\max }}{m}\right)=O\left(d^{\lambda-\kappa}\left|E_{m}\right|^{-1}\right) .
$$


The capacity of the open edges is $d^{(\lambda-\kappa) / 2}\left|E_{m}\right|^{-1}$ so the ratio of the flow calculated above to the edge-capacity is $O\left(d^{(\lambda-\kappa) / 2}\right)$. So, for large enough $d$, the smoothing can be accomplished within $\mathcal{B}$.

We have thus shown that by conditioning on $\mathcal{B}_{\delta}$ being a specific network $\mathcal{B}$ with the properties given (essentially that $T^{2}$ is small) we achieve the required flows with failure probability $O\left(e^{-d^{2}}\right)$ and we have also shown that $\mathcal{B}_{\delta}$ has these properties with failure probability $O\left(e^{-d^{2}}\right)$. Thus we can achieve the flows we require in $\mathcal{B}_{\delta}$ with failure probability $O\left(e^{-d^{2}}\right)$.

We now prove the lemma that gives us a near balanced flow across a single layer. We consider a bipartite network with vertex classes corresponding to $V_{m-1}$ and $V_{m}$ where each edge of $Q^{d}$ is present independently with probability $p$. Edges are given capacity $(1+\epsilon)\left|E_{m}\right|^{-1}$ so that, with high probability, the total capacity is close to $(1+\epsilon) p$. We then introduce a balanced flow of volume $p$ into $V_{m-1}$ and extract a balanced flow of volume $p$ from $V_{m}$ and show that, with high probability, the resultant flow in the network is feasible except for very small imbalances.

Lemma 15. Fix $\epsilon>0$ and suppose that $0<p<1$ and $\ell+1 \leq m \leq d-\ell$. Let $G_{m}$ be the bipartite subgraph induced in $Q^{d}$ with vertex classes $V_{m-1}$ and $V_{m}$, and let $\mathcal{B}_{m}$ be the network formed by picking edges of $G_{m}$ independently with probability $p$ and giving them capacity $(1+\epsilon)\left|E_{m}\right|^{-1}=(1+\epsilon)\left(m\left(\begin{array}{l}d \\ m\end{array}\right)\right)^{-1}$. Then, with failure probability $O\left(e^{-d^{2}}\right)$, there exists a $\left(d^{-3} / 9\right)$-near-balanced feasible flow of volume $p$ from $V_{m-1}$ to $V_{m}$ in $\mathcal{B}_{m}$.

Proof. Choose $p^{\prime}$ with $\max \left\{\frac{p}{1+p}, \frac{p}{1+\epsilon}\right\} \leq p^{\prime}<p$ and define $\delta$ by $(1-p)=$ $\left(1-p^{\prime}\right)(1-\delta)$. Choose $\lambda$ with $1 / 2<\lambda<\kappa$. We then define the networks $\mathcal{B}^{\prime}, \mathcal{B}_{\delta}$ and $\mathcal{B}^{*}$ as follows. $\mathcal{B}^{\prime}$ is formed by picking edges of $G_{m}$ independently with probability $p^{\prime}$ and giving them capacity $\frac{p}{p^{\prime}}\left|E_{m}\right|^{-1}$. $\mathcal{B}_{\delta}$ is formed by picking edges of $G_{m}$ independently with probability $\delta$ and giving them capacity $d^{(\lambda-\kappa) / 2}\left|E_{m}\right|^{-1}$. Next, we let $\mathcal{B}^{*}$ be the network formed by superposing $\mathcal{B}^{\prime}$ and $\mathcal{B}_{\delta}$. Finally we let $\mathcal{B}$ be the network formed by giving an edge-capacity $(1+\epsilon)\left|E_{m}\right|^{-1}$ if the corresponding edge in $\mathcal{B}^{*}$ has non-zero capacity and capacity zero otherwise. We note that $\mathcal{B}$ has the same distribution as $\mathcal{B}_{m}$ and the capacity of any edge in $\mathcal{B}$ is at least the capacity of the corresponding edge in $\mathcal{B}^{*}$. So to show that flows exist in $\mathcal{B}_{m}$ we can analyse flows in $\mathcal{B}^{*}$.

We define random sets

$$
\begin{aligned}
& T_{m}^{\prime}=\left\{u \in V_{m}: d^{\prime}(u)<p^{\prime} m-d^{\lambda} \text { or } d^{\prime}(v)>p^{\prime} m+d^{\lambda}\right\} \\
& S_{m}^{\prime}=V_{m} \backslash T_{m}^{1}
\end{aligned}
$$


where $d^{\prime}(v)$ is the degree of $v$ in the $p^{\prime}$ network $\mathcal{B}^{\prime}$. We similarly define $T_{m-1}^{\prime}$ and $S_{m-1}^{\prime}$ for vertices in layer $V_{m-1}$.

We now condition on the values of the random sets $T_{m-1}^{\prime}$ and $T_{m}^{\prime}$. So let us pick subsets $T_{m-1} \subset V_{m-1}$ with $\left|T_{m-1}\right| \leq\left|V_{m-1}\right| d^{-99}$, and $T_{m} \subset V_{m}$ with $\left|T_{m}\right| \leq\left|V_{m}\right| d^{-99}$ and condition on the events $T_{m-1}^{\prime}=T_{m-1}$ and $T_{m}^{\prime}=T_{m}$.

We introduce a balanced inflow to $V_{m-1}$ of volume $p$ and demand a balanced outflow from $V_{m}$ of volume $p$, and now define a flow from $V_{m-1}$ to $V_{m}$ as follows.

1. Use the full capacity of every edge in $\mathcal{B}^{\prime}$ to route flow from $V_{m-1}$ to $V_{m}$.

2. For $u \in V_{m}$ the imbalance $\rho(u)$ is given by

$$
\rho(u)=\frac{p}{p^{\prime}} d^{\prime}(u)\left|E_{m}\right|^{-1}-p\left|V_{m}\right|^{-1},
$$

and we make two observations about $\rho(u)$. Firstly,

$$
\frac{p}{p^{\prime}} m p^{\prime}\left|E_{m}\right|^{-1}=p m\left|E_{m}\right|^{-1}=p\left|V_{m}\right|^{-1}, \text { so } \mathbb{E}[\rho(u)]=0 .
$$

Secondly $0 \leq d^{\prime}(u) \leq m$, so

$$
-p\left|V_{m}\right|^{-1} \leq \rho(x) \leq \frac{p}{p^{\prime}} m\left|E_{m}\right|^{-1}-p\left|V_{m}\right|^{-1}=\left(\frac{p}{p^{\prime}}-p\right)\left|V_{m}\right|^{-1},
$$

Since $p / p^{\prime} \leq 1+p$ we have $|\rho(x)| \leq\left|V_{m}\right|^{-1}$.

For $u \in V_{m-1}$ the imbalance $\rho(u)$ is given by

$$
\rho(u)=-\frac{p}{p^{\prime}} d^{\prime}(u)\left|E_{m-1}\right|^{-1}+p\left|V_{m-1}\right|^{-1},
$$

and we make the same two observations as above. Namely, $\mathbb{E}[\rho(u)]=0$ and $|\rho(u)| \leq\left|V_{m-1}\right|^{-1}$.

3. Let $S_{m}=V_{m} \backslash T_{m}$ and $S_{m-1}=V_{m-1} \backslash T_{m-1}$. We define $\psi(x)$ as follows:

$$
\psi(x)= \begin{cases}\rho(x)-\left|S_{m}\right|^{-1} \sum_{v \in S_{m}} \rho(v) & \text { if } x \in S_{m} \\ 0 & \text { if } x \in T_{m} \\ \rho(x)-\left|S_{m-1}\right|^{-1} \sum_{v \in S_{m-1}} \rho(v) & \text { if } x \in S_{m-1} \\ 0 & \text { if } x \in T_{m-1} .\end{cases}
$$

For each vertex $x$, flows of volume $\psi(x)$ are now put into $\mathcal{B}_{\delta}$ and 'smoothed' using Lemma 14. The quantities $\psi(x)$ and the network $\mathcal{B}_{\delta}$ satisfy the conditions of Lemma 14 so the smoothing process takes the input $\psi(x)$ and results in output $\theta(x)$, and the resulting imbalance after smoothing at each vertex is $\phi(x)=\rho(x)-\psi(x)+\theta(x)$. 
So

$$
\begin{aligned}
\sum_{x \in V}|\phi(x)| & \leq \sum_{x \in V}|\rho(x)-\psi(x)|+\sum_{x \in V}|\theta(x)| \\
& \leq \sum_{x \in T_{m}}|\rho(x)-\psi(x)|+\sum_{x \in T_{m-1}}|\rho(x)-\psi(x)| \\
& +\sum_{x \in S_{m}}|\rho(x)-\psi(x)|+\sum_{x \in S_{m-1}}|\rho(x)-\psi(x)|+\sum_{x \in V}|\theta(x)| .
\end{aligned}
$$

We are able to achieve a small total for this expression because $|\rho(x)-\psi(x)|$ is small on $T_{m} \cup T_{m-1}$ which is a small set and because $|\rho(x)-\psi(x)|$ is very small on $S_{m} \cup S_{m-1}$. Formally for $x \in T_{m}, \psi(x)=0$ and $|\rho(x)| \leq\left|V_{m}\right|^{-1}$; and for $x \in T_{m-1}, \psi(x)=0$ and $|\rho(x)| \leq\left|V_{m-1}\right|^{-1}$. For $x \in S_{m}, \rho(x)-\psi(x)=$ $\left|S_{m}\right|^{-1} \sum_{v \in S_{m}} \rho(v)$; and for $x \in S_{m-1}, \rho(x)-\psi(x)=\left|S_{m-1}\right|^{-1} \sum_{v \in S_{m-1}} \rho(v)$.

So by equation 6.2 .

$$
\sum_{x \in V}|\phi(x)| \leq \frac{\left|T_{m}\right|}{\left|V_{m}\right|}+\frac{\left|T_{m-1}\right|}{\left|V_{m-1}\right|}+\left|\sum_{v \in S_{m}} \rho(v)\right|+\left|\sum_{v \in S_{m-1}} \rho(v)\right|+\sum_{x \in V}|\theta(x)| .
$$

We now look at each of these terms in turn. By our conditioning $\left|T_{m}\right|\left|V_{m}\right|^{-1}<d^{-99}$ and $\left|T_{m-1}\right|\left|V_{m-1}\right|^{-1}<d^{-99}$, and

$$
\begin{aligned}
\left|\sum_{v \in S_{m}} \rho(v)\right| & =\left|\sum_{v \in V_{m}} \rho(v)-\sum_{v \in T_{m}} \rho(v)\right|, \\
& \leq\left|\sum_{v \in V_{m}} \rho(v)\right|+\left|T_{m}\right|\left|V_{m}\right|^{-1}, \\
& \leq\left|\sum_{v \in V_{m}} \rho(v)\right|+d^{-99} .
\end{aligned}
$$

From Lemma 4 we have

$$
\mathbb{P}\left[\left|\sum_{v \in V_{m}} \rho(v)\right|>d^{-99} p\right] \leq 2 e^{-d^{-198} p^{2}\left(\begin{array}{c}
d \\
m
\end{array}\right) / 2}=O\left(e^{-d^{2}}\right),
$$

and

$$
\mathbb{P}\left[\left|\sum_{v \in V_{m}-1} \rho(v)\right|>d^{-99} p\right] \leq 2 e^{-d^{-198} p^{2}\left(\begin{array}{c}
d \\
m-1
\end{array}\right) / 2}=O\left(e^{-d^{2}}\right) .
$$


From Lemma 14 we get

$$
\mathbb{P}\left[\sum_{v \in V_{m}}|\theta(v)|>d^{-4}\right]=O\left(e^{-d^{2}}\right) .
$$

Putting these expressions back into equation (6.3), subject to the initial conditioning, with failure probability $O\left(e^{-d^{2}}\right)$, we get that $\sum_{x \in V}|\phi(x)|<$ $2 d^{-4}$, which is at most $d^{-3} p / 7$, for large $d$.

It now remains to show that, with high probability, our network $\mathcal{B}$ satisfies $\left|T_{m}^{1}\right| \leq\left|V_{m}\right| d^{-99}$ and $\left|T_{m-1}^{1}\right| \leq\left|V_{m-1}\right| d^{-99}$.

For $x \in V_{m}, d^{\prime}(x)$ is a random variable with distribution $\operatorname{Bin}\left(m, p^{\prime}\right)$ so by taking Chernoff bounds (Lemma $40 \mathbb{P}\left[x \in T_{m}^{1}\right]<e^{-d^{2 \lambda} / 2 m}=e^{-\Omega\left(d^{2 \lambda-1}\right)}$. The events $x \in T_{m}^{1}$ for $x \in V_{m}$ are independent so we can use Lemma 13 to show $\mathbb{P}\left[\left|T_{m}^{1}\right| \geq\left|V_{m}\right| d^{-99}\right]=O\left(e^{-d^{2}}\right)$. So with failure probability $O\left(e^{-d^{2}}\right)$, $\left|T_{m}^{1}\right|\left|V_{m}\right|^{-1} \leq d^{-99}$. Similarly, with failure probability $O\left(e^{-d^{2}}\right)$, we have $\left|T_{m-1}^{1}\right|\left|V_{m-1}\right|^{-1} \leq d^{-99}$. This proves the lemma.

We note that we require $\lambda>1 / 2$ and hence $\kappa>1 / 2$ for this proof to work. In essence the proof would not work if the disparity between the sizes of the two vertex layers was too large.

Next we are going to prove a general result about flows in directed networks. We will use the definitions of a proper $S T$ flow $f$ and its volume $\operatorname{vol}(f)$ from Section 2 .

Lemma 16. Let $\mathcal{N}=(V, \vec{E})$ be a directed network and let $S$ and $T$ be disjoint non-empty subsets of $V$. Let $0<\theta<1 / 9$ and let $f: \vec{E} \rightarrow[0, \infty)$ be a possibly improper flow with the following properties:

$$
\begin{aligned}
& \sum_{v \in V \backslash(S \cup T)}\left|f^{+}(v)\right| \leq \theta \operatorname{size}(f), \\
& \sum_{v \in S}\left|f^{+}(v)-\operatorname{size}(f) /\right| S||+\sum_{v \in T}\left|f^{-}(v)-\operatorname{size}(f) /\right| T|| \leq \theta \operatorname{size}(f),
\end{aligned}
$$

where $\operatorname{size}(f)=1 / 2 \sum_{v \in V}\left|f^{+}(v)\right|$. Then there is a proper ST flow $g$ such that the following hold:

1. For all $\vec{e} \in \vec{E}, 0 \leq g(\vec{e}) \leq f(\vec{e})$.

2. For all $s \in S, g^{+}(s) \geq 0$ and for all $t \in T, g^{-}(t) \geq 0$, and $\operatorname{vol}(g)=$ $\sum_{s \in S} g^{+}(s)=\sum_{t \in T} g^{-}(t) \geq(1-2 \theta) \operatorname{size}(f)$.

3. $\sum_{v \in S}\left|g^{+}(v)-\operatorname{vol}(g) /\right| S||+\sum_{v \in T}\left|g^{-}(v)-\operatorname{vol}(g) /\right| T|| \leq 9 \theta \operatorname{vol}(g)$. 
Proof. We define

$$
\begin{aligned}
V^{+} & =\left\{v \in V \backslash(S \cup T): f^{+}(v)>0\right\}, \\
V^{-} & =\left\{v \in V \backslash(S \cup T): f^{+}(v)<0\right\}, \\
S^{+} & =\left\{s \in S: f^{+}(s)>0\right\} \text { and } S^{-}=\left\{s \in S: f^{+}(s)<0\right\}, \\
T^{+} & =\left\{t \in T: f^{+}(t)>0\right\} \text { and } T^{-}=\left\{t \in T: f^{+}(t)<0\right\} .
\end{aligned}
$$

And we introduce a 'super-source' vertex $x$, and edges $\vec{E}^{+}=\left\{\overrightarrow{x v}: v \in V^{+}\right\}$, $\vec{E}_{S}^{+}=\left\{\overrightarrow{x s}: s \in S^{+}\right\}, \vec{E}_{T}^{+}=\left\{\overrightarrow{x t}: t \in T^{+}\right\}$and a 'super-sink' vertex $y$ and edges $\vec{E}^{-}=\left\{\overrightarrow{v y}: v \in V^{-}\right\}, \vec{E}_{S}^{-}=\left\{\overrightarrow{s y}: s \in S^{-}\right\}$and $\vec{E}_{T}^{-}=\left\{\overrightarrow{t y}: t \in T^{-}\right\}$. (see Figure 3). We now define $f^{\prime}$ by

$$
f^{\prime}(\vec{e})= \begin{cases}f(\vec{e}) & \text { if } \vec{e} \in \vec{E}, \\ f^{+}(v) & \text { for } \vec{e}=x v \in \vec{E}^{+} \cup \vec{E}_{S}^{+} \cup \vec{E}_{T}^{+}, \\ f^{-}(v) & \text { for } \vec{e}=v y \in \vec{E}^{-} \cup \vec{E}_{S}^{-} \cup \vec{E}_{T}^{-}\end{cases}
$$

The new flow $f^{\prime}$ is a proper $x y$ flow on the enlarged network. Also $\operatorname{vol}\left(f^{\prime}\right)=$ $\sum\left\{f^{+}(v): v \in V\right.$ with $\left.f^{+}(v)>0\right\}=\operatorname{size}(f)$. We can decompose this flow into flows along $x y$ paths and around cycles (see for example Ahuja, Magnanti and Orlin [1, page 80]). We then define a new proper $x y$ flow by deleting all the $x y$ flows along paths in this decomposition that use edges in $\vec{E}^{+} \cup \vec{E}_{T}^{+} \cup \vec{E}^{-} \cup \vec{E}_{S}^{-}$. The sum of the volumes of the deleted $x y$ flows is at most

$$
\sum_{v \in V \backslash(S \cup T)}\left|f^{+}(v)\right|+\sum_{v \in S^{-}}\left|f^{-}(v)\right|+\sum_{v \in T^{+}}\left|f^{+}(v)\right| .
$$

Now these last two terms sum to less than $\sum_{v \in S}\left|f^{+}(v)-\operatorname{size}(f) /\right| S||+$ $\sum_{v \in T}\left|f^{-}(v)-\operatorname{size}(f) /\right| T||$ which is less than $\theta \operatorname{size}(f)$.

So the total volume of the deleted flows is at most $2 \theta \operatorname{size}(f)$ and the resultant flow is a proper $x y$ flow with volume at least $(1-2 \theta) \operatorname{size}(f)$ in which every constituent flow starts $x s$ and ends $t y$ for some vertices $s \in S, t \in T$. We now define our proper $S T$ flow $g$ by restricting this flow to the edges in $\mathcal{N}$, i.e. the $s t$ portions of these paths.

By construction $0 \leq g(\vec{e}) \leq f(\vec{e})$ for all $\vec{e} \in \vec{E}$ and $g^{+}(s) \geq 0$ for all $s \in S$ and $g^{-}(t) \geq 0$ for all $t \in T$ and we have just shown $\operatorname{vol}(g)=$ $\sum_{s \in S} g^{+}(s)=\sum_{t \in T} g^{-}(t) \geq(1-2 \theta) \operatorname{size}(f)$. Lastly,

$$
\begin{aligned}
\sum_{v \in S}\left|g^{+}(v)-\operatorname{vol}(g) /\right| S|| & \leq \sum_{v \in S}\left|g^{+}(v)-f^{+}(v)\right|+\sum_{v \in S}\left|f^{+}(v)-\operatorname{size}(f) /\right| S|| \\
& +\sum_{v \in S}|\operatorname{size}(f) /| S|-\operatorname{vol}(g) /| S|| .
\end{aligned}
$$


And the last term equals $|\operatorname{size}(f)-\operatorname{vol}(g)| \leq 2 \theta \operatorname{size}(f)$. So

$$
\begin{aligned}
\sum_{v \in S}\left|g^{+}(v)-\operatorname{vol}(g) /\right| S|| & +\sum_{v \in T}\left|g^{-}(v)-\operatorname{vol}(g) /\right| T|| \leq \sum_{v \in S \cup T}\left|f^{+}(v)-g^{+}(v)\right| \\
& +\sum_{v \in S}\left|f^{+}(v)-\operatorname{size}(f) /\right| S||+\sum_{v \in T}\left|f^{-}(v)-\operatorname{size}(f) /\right| T|| \\
& +|S|^{-1} \sum_{v \in S}|\operatorname{size}(f)-\operatorname{vol}(g)|+|T|^{-1} \sum_{v \in T}|\operatorname{size}(f)-\operatorname{vol}(g)| \\
& \leq 7 \theta \operatorname{size}(f) \leq 9 \theta \operatorname{vol}(g) .
\end{aligned}
$$

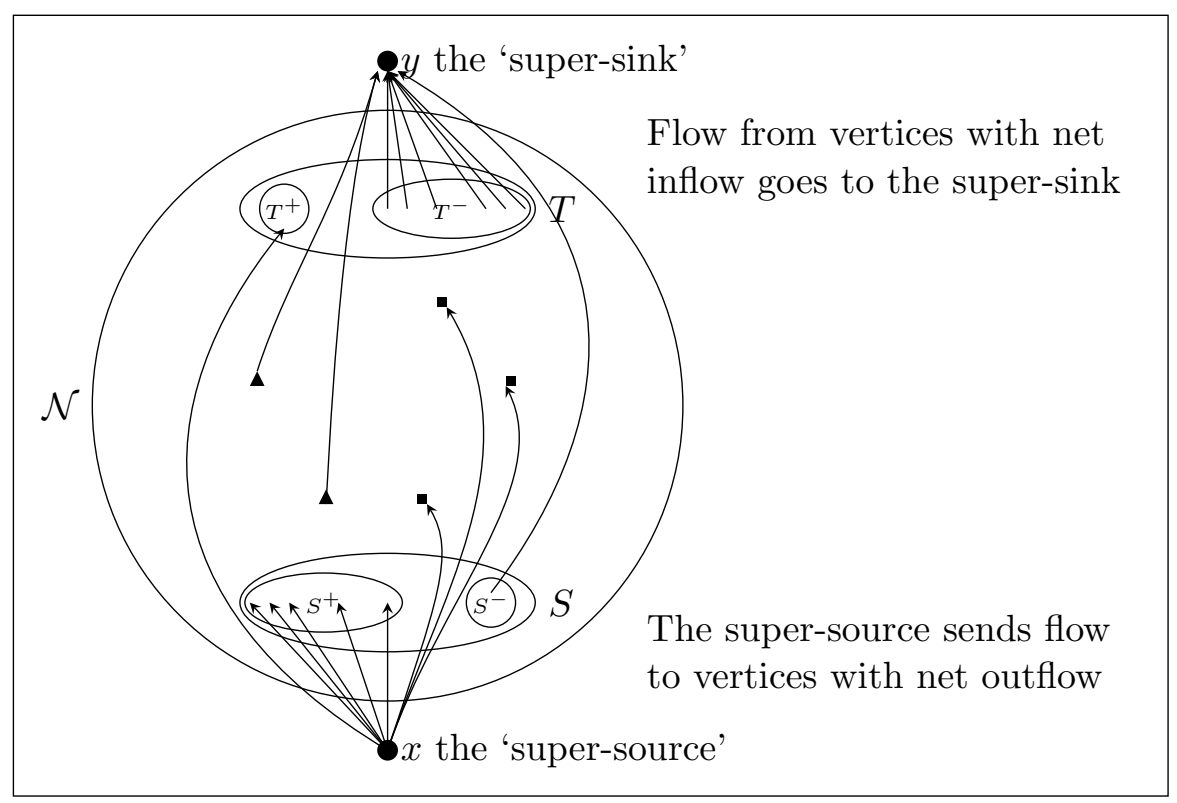

Figure 3: Flows added from a super-source and to a super-sink to create a proper flow.

Lemma 17. Fix $0<p<1$, and $f i x \epsilon>0$. For $\mathcal{N} \in \mathcal{G}\left(Q^{d}, p\right)$, with failure probability $e^{-\Omega\left(d^{2}\right)}$ there exists a $d^{-2}$-near-balanced flow of volume at least $(1+\epsilon)^{-1} p$ from $V_{\ell}(\mathbf{0})$ to $V_{\ell}(\mathbf{1})$ in the network $\mathcal{N}(\mathbf{0})$.

Proof. The proof follows by applying Lemma 15 to each layer from layer $\ell+1$ to layer $d-\ell$. With failure probability $O\left(e^{-d^{2}}\right)$ there is in each layer a $\left(d^{-3} / 9\right)$-near-balanced flow of volume $p$ that satisfies the capacity constraints. By taking the union bound we see that with failure probability $e^{-\Omega\left(d^{2}\right)}$, there 
is an improper flow $F$ of volume $p$ from $V_{\ell}$ to $V_{d-\ell}$ with $\sum\left|f^{+}(v)\right| \leq d^{-2} p / 9$ where the sum is taken over all vertices in layers $m$ to $d-\ell-1$, and which satisfies the capacity constraints of $\mathcal{N}$. By applying Lemma 16 there is a feasible flow of volume $\left(1-d^{-2}\right) p$ from $V_{\ell}$ to $V_{d-\ell}$, which is $d^{-2}$-nearbalanced.

The main result of this section is the following lemma which is the generalization of Lemma 17 to general edge-capacities.

Lemma 18. Let the non-negative random variable $C$ have finite mean. Fix $\epsilon>0$. For $\mathcal{N} \in \mathcal{G}\left(Q^{d}, C\right)$, with failure probability $e^{-\Omega\left(d^{2}\right)}$, the network $\mathcal{N}(\mathbf{0})$ has a $d^{-2}$-near-balanced flow of volume at least $(1-\epsilon) \mathbb{E}[C]$ from $V_{\ell}(\mathbf{0})$ to $V_{\ell}(\mathbf{1})$.

Proof. Lemma 17 gave the result we desire in a network $\mathcal{N} \in \mathcal{G}\left(Q^{d}, p\right)$. We now look at $\mathcal{N} \in \mathcal{G}\left(Q^{d}, C\right)$ for the general distribution $C$. We say a random variable $C$ and its distribution are good if, with failure probability $e^{-\Omega\left(d^{2}\right)}$, there exists a $d^{-2}$-near-balanced flow of volume at least $(1-\epsilon) \mathbb{E}[C]$ from $V_{\ell}(\mathbf{0})$ to $V_{\ell}(\mathbf{1})$ in network $\mathcal{N}(\mathbf{0})$ where the independent edge-capacities have distribution $C$. So we wish to prove that $C$ is good for general distribution $C$ with $\mathbb{E}[C]<\infty$.

Firstly consider a distribution of the form

$$
\mathbb{P}\left[C=a_{i}\right]=p_{i}, 0 \leq i \leq n, \sum_{i=0}^{n} p_{i}=1,0=a_{0}<a_{1}<a_{2} \cdots<a_{n}
$$

where $p_{i}>0$ for $i>0$. Now define $C_{i}=\mathbf{1}_{C=a_{i}}$ and note that $C=\sum_{i=0}^{n} a_{i} C_{i}$ and $C_{i} \sim \operatorname{Ber}\left(p_{i}\right)$. Now consider a network $\mathcal{N}^{\prime}$ generated by superimposing networks formed with edge-capacities $a_{i} C_{i}$ and note that $\mathcal{N}^{\prime}$ is a valid generator of $\mathcal{N}$. Now a scaled distribution of a good distribution is good and a finite sum of (not necessarily independent) good distributions is also good. To see this, as $\epsilon>0$, we know, with failure probability $e^{-\Omega\left(d^{2}\right)}$, there exists a $d^{-2}$-near-balanced flow of volume at least $(1-\epsilon) \mathbb{E}\left[a_{i} C_{i}\right]$ in the network formed with edge-capacities $a_{i} C_{i}$. By taking the union bound we know that the sum of these flows gives a flow of volume at least $(1-\epsilon) \mathbb{E}\left[\sum_{i} a_{i} C_{i}\right]$ with failure probability $e^{-\Omega\left(d^{2}\right)}$. The volume of the sum of these flows equals the sum of the volumes of the individual flows so the resultant flow is also $d^{-2}$-near-balanced.

Hence all $C$ of the form $(6.4)$ are good. For general distribution $C$ with $\mathbb{E}[C]<\infty$ and $\epsilon>0$ we can truncate and approximate $C$ by a random variable $C^{(\epsilon)}$ of the form (6.4) such that $\mathbb{E}\left[C^{(\epsilon)}\right]>\mathbb{E}[C]-\epsilon$ and $C \geq C^{(\epsilon)}$. 


\section{Proofs of Lemma 6 and Theorem 1}

\subsection{Proof of Lemma 6}

In Lemma 12 we showed that given $\epsilon>0$ there exists a constant $M$ such that, with failure probability $O\left(2^{-\rho d}\right)$ we can route a balanced flow of volume $\mathbb{E}[C]$ in the network $\mathcal{N}^{M}(u)$ between $u$ and layer $V_{\ell}(u)$ for each vertex $u$ in $Q^{d}$. Let us condition on this happening and consider vertex $\mathbf{0}$. In Lemma 18 we showed that with failure probability $e^{-\Omega\left(d^{2}\right)}$ we could route a $d^{-2}$-nearbalanced flow of volume at least $(1+\epsilon)^{-1} \mathbb{E}[C]$ from $V_{\ell}(\mathbf{0})$ to $V_{d-\ell}(\mathbf{0})$. By Lemma 16 these can be combined to give a flow from $\mathbf{0}$ to $\mathbf{1}$ of volume at least $(1+\epsilon)^{-1} \mathbb{E}[C]-d^{-2}$. The edges sets used by the three sets of flows defined above only overlap in $E_{\ell+1}, E_{\ell+2}, E_{d-\ell-1}, E_{d-\ell}$ and the sum of the capacities used is less than the capacity in $\mathcal{N}^{M+1}(\mathbf{0})$. Thus for large enough $d$ and by a suitable redefinition of $\epsilon$ we see that subject to our conditioning with failure probability $e^{-\Omega\left(d^{2}\right)}$ we can route a balanced flow of volume $(1-\epsilon) \mathbb{E}[C]$ in the network $\mathcal{N}^{M+1}(\mathbf{0})$ between $\mathbf{0}$ and $\mathbf{1}$. We can remove the condition to prove the lemma.

\subsection{Proof of Theorem 1}

The upper bound (1.1) has already been proved in Section 3 so it remains to prove the lower bound $(1.2)$. Lemma 6 showed that given $\epsilon>0$ there exists a constant $M$ such that, with failure probability $O\left(2^{-\rho d}\right)$, for each antipodal vertex pair $u, \bar{u}$, there exists a flow of volume $(1-\epsilon) \mathbb{E}[C]$ between $u$ and $\bar{u}$ in the network $\mathcal{N}^{M}(u)$. We now consider the flows of all antipodal vertex pairs simultaneously in the network $\mathcal{N}_{\text {sum }}$ which is formed by superposing the networks $\mathcal{N}^{M}(u)$ for all vertex pairs. The lower bound in Theorem 1 is proved by showing in Lemma 19 that the capacity demanded of an edge $e$ in $\mathcal{N}_{\text {sum }}$ is less than $(1+o(1)) c_{e}$ and then rescaling.

The capacity $\operatorname{cap}(e)$ of edge $e$ required in $\mathcal{N}_{\text {sum }}$ is the sum of the capacity required in that edge for all antipodal vertex pairs. Then

$$
\operatorname{cap}(e)=\frac{1}{2} \sum_{v \in V} \mathbf{1}_{e \in E_{m}(v)} \frac{c_{e} M(m)}{\left|E_{m}\right|},
$$

where $M(m)=M$ for $1 \leq m \leq \ell+2$ and for $d-\ell-1 \leq m \leq d$ and $M(m)=1$ otherwise, and $\mathbf{1}_{e \in E_{m}(v)}$ is an indicator function that takes the value 1 when $v$ is a vertex for which the edge $e$ is in edge-layer $m$. For ease 
of calculation we write this out as follows.

$$
\begin{aligned}
\operatorname{cap}(e)=\sum_{m=1}^{d} \sum_{v: e \in E_{m}(v)} \frac{c_{e}}{2\left|E_{m}\right|} & +\sum_{m=1}^{\ell+2} \sum_{v: e \in E_{m}(v)} \frac{c_{e}(M-1)}{2\left|E_{m}\right|} \\
& +\sum_{m=d-\ell-1}^{d} \sum_{v: e \in E_{m}(v)} \frac{c_{e}(M-1)}{2\left|E_{m}\right|} .
\end{aligned}
$$

Lemma 19. Fix an edge $e$ in $Q$ and let $\epsilon_{d}=\frac{2(M-1)(\ell+2)}{d}$, and $V=V(Q)$. Then
1. $\sum_{m=1}^{d} \sum_{v: v \in E_{m}(v)} \frac{c_{e}}{\left|E_{m}\right|}=2 c_{e}$,
2. $\sum_{m=1}^{d} \sum_{v: v \in E_{m}(v)} \frac{c_{e}(M-1)}{\left|E_{m}\right|}=2 \epsilon_{d} c_{e}$,
3. $\operatorname{cap}(e)=\left(1+\epsilon_{d}\right) c_{e}$.

Proof. A vertex $v$ has $\left|E_{m}\right|$ edges in edge-layer $E_{m}(v)$ so in the cube there are $|V(Q)|\left|E_{m}\right|$ vertex-edge pairs where the edge is in layer $m$ of the vertex. Therefore, by the symmetry of the cube, each edge has $\left|E_{m}\right| \frac{|V(Q)|}{|E(Q)|}=2\left|E_{m}\right| / d$ vertices for which it is in edge-layer $m$. So

$$
\sum_{m=1}^{d} \sum_{v: e \in E_{m}(v)} c_{e}\left|E_{m}\right|^{-1}=\sum_{m=1}^{d} \frac{2 c_{e}\left|E_{m}\right|}{d}\left|E_{m}\right|^{-1}=2 c_{e}
$$

Similarly,

$$
\begin{aligned}
& \sum_{m=1}^{\ell+2} \sum_{v: e \in E_{m}(v)} c_{e}(M-1)\left|E_{m}\right|^{-1}+\sum_{\substack{m=\\
d-\ell-1}}^{d} \sum_{v: e \in E_{m}(v)} c_{e}(M-1)\left|E_{m}\right|^{-1} \\
= & (M-1) \sum_{m=1}^{\ell+2} c_{e} \frac{2\left|E_{m}\right|}{d}\left|E_{m}\right|^{-1}+(M-1) \sum_{\substack{m=\\
d-\ell-1}}^{d} c_{e} \frac{2\left|E_{m}\right|}{d}\left|E_{m}\right|^{-1} \\
= & 4 \frac{(M-1)(\ell+2)}{d} c_{e}=2 \epsilon_{d} c_{e} .
\end{aligned}
$$

The third assertion follows by adding together the capacities from the two parts above. 


\section{Proof of Theorem 2}

\subsection{Overview}

In Theorem 2 we consider flows between all vertex pairs in the network $\mathcal{N} \in \mathcal{G}\left(Q^{d}, C\right)$. We introduce the following terminology. For $u, v \in V\left(Q^{d}\right)$ we denote by $Q(u, v)$ the smallest cube containing both $u$ and $v$. If $d_{Q}(u, v)=k$ then $Q(u, v)$ has $2^{k}$ vertices. In set notation the vertices of $Q(u, v)$ are those subsets of $u \cup v$ which contain $u \cap v$. For $u, v \in V\left(Q^{d}\right)$ with $d(u, v)=k$ we denote by $\mathcal{N}^{M}(u, v)$ the subcube $Q(u, v)$ with edge-capacities defined in a manner analogous to equation (4.1). For edge $e$ in edge-layer $m$ in $Q(u, v)$, we let $c_{e}=2^{1-d}\left(\begin{array}{c}k \\ m\end{array}\right)^{-1} c_{e} m^{-1}$ for $\ell+3 \leq m \leq k-\ell-2$ and $c_{e}=M 2^{1-d}\left(\begin{array}{c}k \\ m\end{array}\right)^{-1} c_{e} m^{-1}$ for $m \leq \ell+2$ or $m \geq k-\ell-1$. We let $S_{u}(v)=Q(u, v) \cap V_{\ell}(u)$. See figure 4. We consider vertex pairs with a small separation separately in Section 8.5. So let $\mathcal{V}^{\text {near }}$ denote the set of unordered pairs of vertices $u, v$ such that $d(u, v) \leq d / 4$ and let $\mathcal{V}^{\text {far }}=\mathcal{V} \backslash \mathcal{V}^{\text {near }}$.

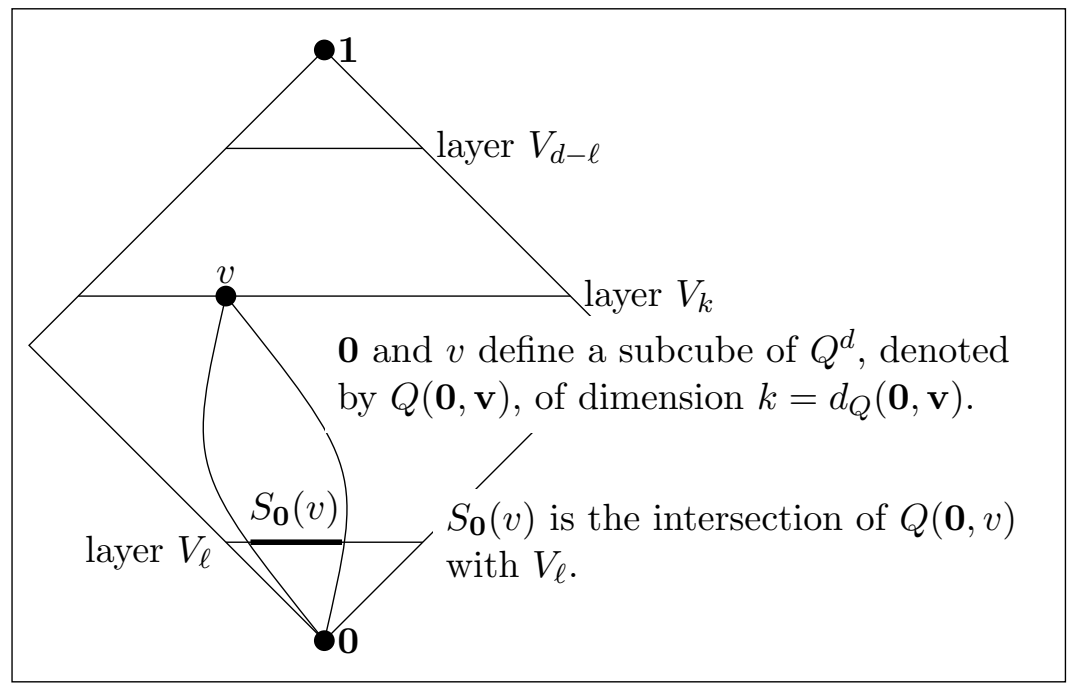

Figure 4: The $k$-cube defined by $\mathbf{0}$ and $v$ and the set $S_{\mathbf{0}}(v)$

The proof of Theorem 2 uses much of the work from the first proof but we must be careful about how we show that we can escape from every vertex. For example if $d$ is even and we want to route a flow from $u$ to $v$ with $d_{Q}(u, v)=d / 2$ we cannot just apply theorem 1 to $Q(u, v)$ as the failure probability in Lemma 12 is too great. This is not just a technical problem: if the open degree of $u$ is less than $d / 2$ then there is some vertex $v$ with $d_{Q}(u, v)=d / 2$ such that $u$ is an isolated vertex in $Q(u, v)$. However, by Lemma 12 we know that for some constant $M$, with high probability, we can 
route a balanced flow to $V_{\ell}$ in $\mathcal{N}^{M}(u)$. Lemma 20 is a deterministic result that uses the symmetry of the cube to divide up this flow and allocate the parts to the different flows from $u$ to all the sets $S_{u}(v)$ in a balanced manner to achieve the flows we need.

In Lemma 22 we show that with high probability we can route $(1+$ $\epsilon)^{-1} d^{-2}$-near-balanced flows of volume $\mathbb{E}[C] 2^{1-d}$ between $S_{u}(v)$ and $S_{v}(u)$ for all $\{u, v\} \in \mathcal{V}^{\text {far }}$. We further show that the capacity required in an edge $e$ to achieve all these flows simultaneously is less than $c_{e}$. We then 'stitch' these flows together using Lemma 16 in the proof of Lemma 23 , which covers flows between all vertex pairs in $\mathcal{V}^{\text {far }}$.

In Lemma 24 we show that for all pairs $\{u, v\} \in \mathcal{V}^{\text {near }}$ we can route simultaneous flows via distant vertices and that the total capacity required for edge $e$ in this network is less than $\epsilon c_{e}$.

The theorem follows from these lemmas.

\subsection{Flows close to a source}

In Lemma 12 we showed that there exist constants $\rho>0$ and $M>0$ such that the following holds. With failure probability $2^{-\rho d}$ :- for all vertices $u$ there exists a balanced flow $f_{u}$ of volume $\mathbb{E}[C]$ in the network $B_{u}^{M}(\ell+2)$ from $u$ to $V_{\ell}(u)$. We further showed in Lemma 19 that for each edge $e$ of $Q$ the sum of the absolute values of the flows along $e$ from the $f_{u}$ is at most $\epsilon_{d} c_{e}$ where $\epsilon_{d}=\frac{2(\ell+2) M}{d}=O\left(d^{\kappa-1}\right)$

We now want to prove the deterministic result that if $\mathcal{N}^{*}$ is a network (on $\left.Q^{d}\right)$ with the above property then for every vertex $u$ we can decompose the flow from $u$ to $V_{\ell}(u)$ into balanced flows of volume $2^{-d}$ from $u$ to $S_{u}(v)$ for all $v \in V_{k}$ and all $d / 4<k \leq d$, and that this can be accomplished using only a small $\left(\epsilon_{d}\right)$ proportion of the capacity of any edge. (Note that for presentation purposes we decompose flows of volume 1 from $u$ to $V_{\ell}(u)$ implying a different choice of the constant $M$ ).

Lemma 20. Let $f$ be a balanced flow of volume 1 from $\mathbf{0}$ to $V_{\ell}$ in $Q^{d}$. Then $f$ may be decomposed as $g+\sum_{v} f^{v}$ where the sum is over $v \in Q^{d}$ with $d(\mathbf{0}, v)>d / 4$ and where $f_{v}$ is a balanced $\mathbf{0}-S_{\mathbf{0}}(v)$ flow of volume $2^{-d}$ and there is no cancellation in the sum.

Proof. Let $\mathbf{0} \in V\left(Q^{d}\right)$, and let $f^{\mathbf{0}}$ be a balanced flow of volume 1 in $B_{\ell+2}^{* M}(\mathbf{0})$ from $\mathbf{0}$ to $V_{\ell}(\mathbf{0})=V_{\ell}$. We first show that there are flows $f^{\mathbf{0} v}$ for each vertex $v$ such that $\{\boldsymbol{0}, v\} \in \mathcal{V}^{\text {far }}$ such that i) $f^{\mathbf{0} v}$ is a balanced flow of volume $2^{-d}$ from $\mathbf{0}$ to $S_{\mathbf{0}}(v)$, and that ii) $\sum_{v:\{u, v\} \in \mathcal{V}^{\text {far }}}\left|f^{\mathbf{0} v}\right| \leq f^{\mathbf{0}}(e)$ for each edge $e \in E\left(Q^{d}\right)$.

The flow $f^{0}$ can be decomposed into flows along paths and around cycles (see for example [1]). For each $w \in V_{\ell}(\mathbf{0})$ we denote by $F_{w}$ the sum of all 
the flows along $\mathbf{0} w$ paths that end at vertex $w . F_{w}$ is therefore a flow of volume $\left|V_{\ell}\right|^{-1}$ from $\mathbf{0}$ to $w$. For $d / 4<k \leq d$ and $v \in V_{k}(\mathbf{0})$ and for each $w \in S_{\mathbf{0}}(v)$ we now allocate a flow of volume $\left(\begin{array}{l}k \\ l\end{array}\right)^{-1} 2^{-d}$ out of the total flow $F_{w}$ as commodity $\mathcal{K}_{\mathbf{0}, v} \cdot\left|S_{\mathbf{0}}(v)\right|=\left(\begin{array}{l}k \\ l\end{array}\right)$ so this flow allocation is a balanced flow of volume $2^{-d}$ from 0 to $S_{\mathbf{0}}(v)$. We now perform this allocation for all $v \in V_{k}$ for all $d / 4<k \leq d$ and we need to check that the total volume of flow allocated is at most the original flow to each vertex in $V_{\ell}(\mathbf{0})$, namely $\left|V_{\ell}\right|^{-1}$.

For a particular $k$, each $w \in V_{\ell}(u)$ is in $\left(\begin{array}{l}d-\ell \\ k-\ell\end{array}\right)$ sets $S_{\mathbf{0}}(v)$ so the total flow required at $w$ is

$$
\begin{aligned}
\sum_{k=\lfloor d / 4\rfloor+1}^{d}\left(\begin{array}{l}
d-\ell \\
k-\ell
\end{array}\right)\left(\begin{array}{l}
k \\
\ell
\end{array}\right)^{-1} 2^{-d} & =\sum_{k=\lfloor d / 4\rfloor+1}^{d}\left(\begin{array}{l}
d \\
k
\end{array}\right)\left(\begin{array}{l}
k \\
\ell
\end{array}\right)\left(\begin{array}{l}
d \\
\ell
\end{array}\right)^{-1}\left(\begin{array}{l}
k \\
\ell
\end{array}\right)^{-1} 2^{-d} \\
& =\left|V_{\ell}\right|^{-1} \sum_{k=\lfloor d / 4\rfloor+1}^{d}\left(\begin{array}{l}
d \\
k
\end{array}\right) 2^{-d} \\
& \leq\left|V_{\ell}\right|^{-1}
\end{aligned}
$$

Hence we have shown that there exists a balanced flow of volume $2^{-d}$ of commodity $\mathcal{K}_{\mathbf{0}, v}$ from $\mathbf{0}$ to $S_{\mathbf{0}}(v)$ in $B_{\ell+2}^{* M}(\mathbf{0})$ for all $v$ in layers $\lfloor d / 4\rfloor+1$ to $d$.

The flow volume in edge $e$ from all flows from $u$ to $S_{u}(v)$ for all $\{u, v\} \in$ $\mathcal{V}^{\text {far }}$ is at most the sum of the capacities of edge $e$ in the balls $B_{m}^{M}(u)$ for all $u$ and all $1 \leq m \leq \ell+2$ which is at most $\epsilon_{d} c_{e}$ by Lemma 19 .

Putting Lemma 12 together with Lemma 20 we get

Lemma 21. Fix $\epsilon>0$ and suppose that $C$ is a random variable with $\mathbb{P}[C>$ $0]>\frac{1}{2}$. Then there exists constants $\rho>0$ and $M>0$ such that the following holds with failure probability $2^{-\rho d}$. For $\mathcal{N} \in \mathcal{G}\left(Q^{d}, C\right)$, for all vertex pairs $\{u, v\} \in \mathcal{V}^{\text {far }}$ there exists a balanced flow of volume $2^{-d}$ from $u$ to $S_{u}(v)$ in $B_{\ell+2}^{M}(u)$, such that the total flow volume (with no cancellation) from all such flows in any edge $e$ in $\mathcal{N}$ is at most $\epsilon c_{e}$.

\subsection{The flow in the middle part of the flow}

Lemma 22. Fix $\epsilon>0$ and suppose that $C$ is a random variable with $\mathbb{P}[C>$ $0]>\frac{1}{2}$ and let $\mathcal{N} \in \mathcal{G}\left(Q^{d}, C\right)$. With failure probability $O\left(e^{-\Omega\left(d^{2}\right)}\right)$, for all $\{u, v\} \in \mathcal{V}^{\text {far }}$ there exist simultaneous $d^{-2}$-near-balanced flows of volume $(1+$ $\epsilon)^{-1} \mathbb{E}[C] 2^{1-d}$ between $S_{u}(v)$ and $S_{v}(u)$ in the network $\mathcal{N}$.

Proof. Lemma 18 showed that with failure probability $e^{-\Omega\left(d^{2}\right)}$ there exists a $d^{-2}$-near-balanced flow of volume $\mathbb{E}[C]$ between $V_{\ell}(\mathbf{0})$ and $V_{\ell}(\mathbf{1})$ in $\mathcal{N}(u)$. 
Applying this result to the networks $\mathcal{N}^{M}(u, v)$ for all vertex pairs $u, v$ with $d(u, v)>d / 4$ and taking the union bound we see that with failure probability $2^{2 d} e^{-\Omega\left(d^{2}\right)}=e^{-\Omega\left(d^{2}\right)}$ there exists simultaneous $d^{-2}$-near-balanced flows of volume $(1+\epsilon)^{-1} \mathbb{E}[C]$ from $S_{u}(v)$ to $S_{v}(u)$ in $\mathcal{N}^{M}(u, v)$ for every vertex pair $u, v \in \mathcal{V}^{\text {far }}$. We now scale these flows by a factor $2^{1-d}$ and superpose these simultaneously in the network $\mathcal{N}$. The capacity required is at most the capacity calculated from the sum of the capacities of all networks $\mathcal{N}^{M}(u, v)$ for all $\{u, v\} \in \mathcal{V}^{\text {far }}$. Consider first all sub-cubes $Q(u, v)$ for which $d(u, v)=k$ (counting sub-cube $Q(u, v)$ and $Q(v, u)$ once). There are $2^{d-1}\left(\begin{array}{l}d \\ k\end{array}\right)$ such subcubes and they each have $\left(\begin{array}{l}k \\ m\end{array}\right)$ edges in edge-layer $m$ from $u$ so each edge is in $\frac{2^{d-1}\left(\begin{array}{l}d \\ k\end{array}\right)\left(\begin{array}{l}k \\ m\end{array}\right)}{d 2^{d-1}}$ such sub-cubes in edge layer $m$. Thus an edge $e$ has to contribute a total capacity at most $\left(\begin{array}{l}d \\ k\end{array}\right)\left(\begin{array}{l}k \\ m\end{array}\right) d^{-1} \frac{2^{1-d} c_{e}}{\left(\begin{array}{c}k \\ m\end{array}\right)}$ to the flows of volume $2^{1-d}(1+\epsilon)^{-1} \mathbb{E}[C]$ in the networks $\mathcal{N}^{M}(u, v)$ with $d_{Q}(u, v)=k$ for which it is in layer $m$. Thus the total capacity required in edge $e$ is at most

$$
\begin{aligned}
\sum_{k>d / 4}^{d} \sum_{m=\ell}^{k-\ell-1}\left(\begin{array}{l}
d \\
k
\end{array}\right)\left(\begin{array}{l}
k \\
m
\end{array}\right) d^{-1} \frac{c_{e} 2^{1-d}}{\left(\begin{array}{l}
k \\
m
\end{array}\right)} & \leq \sum_{k=1}^{d} \sum_{m=1}^{k}\left(\begin{array}{l}
d \\
k
\end{array}\right) 2^{1-d} d^{-1} c_{e} \\
& =2^{1-d} \sum_{k=1}^{d} \frac{k}{d}\left(\begin{array}{l}
d \\
k
\end{array}\right) c_{e} \\
& =2^{1-d} \sum_{k=0}^{d-1}\left(\begin{array}{c}
d-1 \\
k
\end{array}\right) c_{e}=c_{e}
\end{aligned}
$$

\subsection{Flows between vertex pairs with large separation}

Let $\mathcal{N} \in \mathcal{G}\left(Q^{d}, C\right)$ and denote by $\Phi_{\text {far }}$ the maximum uniform flow volume when $\mathcal{V}$ is taken as $\mathcal{V}^{\text {far }}$

Lemma 23. Fix $\epsilon>0$ and suppose that $\mathbb{P}[C>0]>\frac{1}{2}$. Then as $d \rightarrow \infty$,

$$
\mathbb{P}\left[2^{d-1} \Phi_{\text {far }} \geq(1-\epsilon) \mathbb{E}[C]\right] \rightarrow 1 .
$$

Proof. Lemma 21 showed that given $\epsilon>0$ there exists constants $\rho>0$ and $M>0$ such that with failure probability $2^{-\rho d}$ the following holds. There exist simultaneous balanced flows of volume $2^{-d}$ in $B_{u}^{M}(\ell+2)$ between $u$ and $S_{u}(v)$ for all pairs $\{u, v\}$ in $\mathcal{V}^{\text {far }}$ such that the total flow (with no cancellation) from all such flows occurring in any edge $e$ in $\mathcal{N}$ is less than $\epsilon c_{e}$. Lemma 22 showed that with failure probability $e^{-\Omega\left(d^{2}\right)}$ for all vertex pairs $\{u, v\} \in \mathcal{V}^{\text {far }}$ 
there exist simultaneous $d^{-2}$-near-balanced flows of volume $(1+\epsilon)^{-1} \mathbb{E}[C] 2^{1-d}$ between $S_{u}(v)$ and $S_{v}(u)$ in $\mathcal{N}$.

Thus we achieve the flows we require by scaling the flows and capacities from Lemma 21 by $\mathbb{E}[C]$ and 'stitching' them together with the flows from Lemma 22 using the results from Lemma 16. The capacity of edge $e$ in the superimposed networks is shown to be at most $(1+\epsilon) c_{e}$ by Lemma 19 .

\subsection{Vertex pairs with small separation}

To route flows between pairs of vertices that are 'near' $(d(u, v) \leq d / 4)$ we route flows to distant vertices and back again. We have already shown that, w.h.p. these flows exist, and the number of 'near' vertex pairs is small so the flows can be accommodated in a small part of the capacities of any edge.

Lemma 24. Fix $\epsilon>0$ and suppose that $\mathbb{P}[C>0]>\frac{1}{2}$. Let $\mathcal{N} \in \mathcal{G}\left(Q^{d}, C\right)$. Then there exists a constant $\rho>0$ such that with failure probability $2^{-\rho d}$ there are simultaneous flows of volume $2^{1-d} \mathbb{E}[C]$ between vertices $u$ and $v$ for all $\{u, v\} \in \mathcal{V}^{\text {near }}$ in the network $\mathcal{N}$ with the edge-capacities of $\mathcal{N}$ scaled by $\epsilon$.

Proof. We want a flow of volume $2^{1-d} \mathbb{E}[C]$ between two vertices $u, v$ a distance apart less than $d / 4$. The idea is to route half the flow from $u$ to $\bar{u}$ (the antipodal point of $u$ ) and then back to $v$ and half from $u$ to $\bar{v}$ and then back to $v$. From Lemma 23 , with high probability, all of these flows $(u$ to $\bar{u}, \bar{u}$ to $v, v$ to $\bar{v}, \bar{v}$ to $u$ ) with volumes $(1-\epsilon)^{-1} \mathbb{E}[C]$ exist in networks $\mathcal{N}(u, \bar{u}), \mathcal{N}(u, \bar{v})$ for all $\{u, v\}$. The number of such vertex pairs is at most $2^{d}(d / 4)\left|V_{\lfloor d / 4\rfloor}\right| \leq 2^{d} d(4 e)^{d / 4} \leq 2^{1.9 d}$ for large $d$. Hence, by the symmetry of the cube, the total flow in any edge $e$ from all these flows is at most

$$
2 d 2^{1-d}(1-\epsilon)^{-1} \mathbb{E}[C] \frac{2^{1.9 d}}{d 2^{d-1}} \rightarrow 0
$$

\section{Concluding Remarks}

We have investigated uniform multicommodity flows in the cube with random edge-capacities distributed like some given random variable $C$. There are two natural directions for further investigation. Firstly, this paper has been restricted to the case $\mathbb{P}[C>0]>1 / 2$ which ensures that, with high probability, the network $\mathcal{N}$ is connected, and in this case we have been able to tell a full story. The component structure of the network in the case 
$C \sim \operatorname{Ber}(p)$ for $0<p \leq 1 / 2$ is more complicated. (See [17] for a full analysis). Multicommodity flows in the largest component of such networks are investigated in another paper [16]. We remark that for $d \geq 4$ there exists a disconnected subgraph of $Q^{d}$ in which each antipodal pair of vertices is connected by a path. Such a subgraph could support a non-zero antipodal multicommodity flow despite being disconnected. However for $p<1 / 2$, the probability of isolated vertices tends to 1 and hence the probability of a non-zero uniform multicommodity flow tends to zero.

Secondly, this paper looked at undirected networks but it is also interesting to look at directed networks formed by replacing each undirected edge of $Q^{d}$ with a pair of opposingly directed edges with (possibly identical, possibly independent) random edge-capacities. We study this in [21.

\section{References}

[1] R. Ahuja, T. Magnanti and J. Orlin, Network Flows, Prentice Hall, (1993).

[2] D. Aldous, Optimal flow through the disordered lattice, The Annals of Probability 35 (2007), 397-438.

[3] D. Aldous and S. Bhamidi, Edge flows in the complete random-lengths network, Random Structures and Algorithms 37 (2010), 271-311.

[4] D. Aldous, C. McDiarmid and A. Scott, Uniform multicommodity flow through the complete graph with random edge-capacities, Operations Research Letters 37 (2009), 299-302.

[5] N. Alon and J. Spencer, The Probabilistic Method, Wiley, third edition, (2008).

[6] M. Ajtai, J. Komlós and E. Szemerédi, Largest random component of a $k$-cube, Combinatorica 2 (1982), 1-7.

[7] B. Bollobás, Complete matchings in random subgraphs of the cube, Random Structures and Algorithms 1 (1990), 95-104.

[8] B. Bollobás, Y. Kohayakawa and T. Łuczak, The evolution of random subgraphs of the cube, Random Structures and Algorithms 3 (1992), 5590 . 
[9] B. Bollobás, Y. Kohayakawa and T. Łuczak, On the diameter and radius of random subgraphs of the cube, Random Structures and Algorithms $\mathbf{5}$ (1994), 627-648.

[10] B. Bollobás, Y. Kohayakawa and T. Łuczak, Connectivity properties of random subgraphs of the cube, Random Structures and Algorithms 6 (1995), 221-230.

[11] C. Borgs, J. Chayes, R. van der Hofstad, G. Slade and J. Spencer, Random subgraphs of finite graphs: III, The phase transition for the n-cube, Combinatorica 26 (2006), 395-410.

[12] P. Erdös and J. Spencer, Evolution of the $n$-cube, Comp. and Math with Appl. 5 (1979), 33-39.

[13] M. Khandawawala and R. Sundaresan, Optimal multicommodity flow through the complete graph with random edge-capacities, J. Appl. Prob. 47 (2010), 201-215.

[14] T. Leighton, F. Makedon, S. Plotkin, C. Stein, E. Tardos and S. Tragoudas, Fast approximation algorithms for multicommodity flow problems, J.Comp.Syst.Sci 50 (1992), 228-243.

[15] T. Leighton and S. Rao, Multicommodity max-flow min-cut theorems and their use in designing approximation algorithms, Journal of the ACM 46 (1999), 787-832.

[16] C. McDiarmid, A. Scott and P. Withers, Uniform multicommodity flows in the largest component of the hypercube with random edge-capacities, in preparation, 2015.

[17] C. McDiarmid, A. Scott and P. Withers, The component structure of the random hypercube, in preparation, 2015.

[18] A. Sinclair, Improved bounds for mixing rates of Markov chains and multicommodity flow, Combinatorics, Probability and Computing 1 (1992), 351-370.

[19] W. Winston, Operations Research: Applications and Algorithms (4th edn.), Thompson, (2004).

[20] L. Valiant and G.Brebner, Universal schemes for parallel computing, Proceedings of the thirteenth annual ACM symposium on Theory of computing (1981), 262-277. 
[21] P. Withers, Multicommodity flows in random networks, Doctoral thesis, The University of Oxford, (2015). 